\title{
1 Economic impact of persistent sensor and actuator faults in concrete core activated office buildings
}

3 Joachim Verhelst ${ }^{1,2,3, *}$, Geert Van Ham ${ }^{2,4}$, Dirk Saelens ${ }^{1,5}$, Lieve Helsen ${ }^{1,3}$

4 Abstract

5 Sensor and actuator degradation occurs frequently in office buildings. These 6 can have a large impact on HVAC performance, both on energy use and 7 thermal (dis)comfort. The degree of impact depends on the faulty com8 ponent(s), fault type(s) and fault severity and has a significant non-linear 9 relation with the control strategy and comfort constraints. Concrete core 10 activated (CCA) office buildings typically have a high thermal inertia, high 11 comfort requirements and they are equipped with low exergy and low ca-

Email address: Joachim.Verhelst@kuleuven.be (Joachim Verhelst )

${ }^{1}$ EnergyVille: www.energyville.be

${ }^{2}$ KULeuven, FIIW, Technology campus Geel

${ }^{3}$ KULeuven, dept of Mechanical engineering

${ }^{4}$ KULeuven, dept of Electrical engineering

${ }^{5}$ KULeuven, dept of Civil engineering 

44 CCA

most economical, fault-robust controller for a certain building type. Also, the most critical sensors and actuators are identified.

The evaluated faults are shown to be detrimental for the control performance. The relative economic impact of simultaneous (realistic, randomly distributed and non-correlated) sensor and actuator faults, ranged from +7 $\%$ to $+1000 \%$. By adhering to an appropriate commissioning frequency, this impact can be reduced. the optimal commissioning period for sensors and actuators was determined to be between 2.8 and 5.0 years (case study, controller and assumption dependent). The lowest financial impact due to degradation faults, for this case study and assumptions, is attained by the closed loop model predictive control (CL-MPC) supervisory algorithm, which incurred only a $15 \%$ relative increase of total present cost, as opposed to increases above $+100 \%$ for the other investigated control strategies over the controller lifetime.

This study highlights the relevance of taking faults into account when evaluating long term HVAC control performance and quantifies the economic impact of simultaneous persistent sensor and actuator faults on on control performance.

Keywords: HVAC, MPC, errors, persistent bias, Fault impact, Sensitivity, 


\section{Introduction and background}

In modern office buildings, the increased uptake of renewable, moderate temperature technologies lead to ever more dynamic interactions between the different HVAC components. While installation and control settings are often kept unchanged over the building lifetime, many building usage parameters, such as number of occupants, preferences and schedules tend to vary drastically over the office building life cycle.

The control performance of dynamic systems varies significantly with operating conditions, especially if control settings are not tuned correctly to the system dynamics of the controlled plant. Low-level HVAC systems control is usually of the $\mathrm{P}(\mathrm{I}) \mathrm{D}$ type, which can be notoriously sluggish or oscillatory, if badly tuned $(1,2,3)$. Poorly maintained, degraded, and improperly controlled equipment leads to an estimated $15 \%$ to $30 \%$ of extra energy use in commercial buildings (4). There are many different causes for these degradations: building design and construction is a highly competitive, price-driven industry. The application of large safety margins and rules of thumb in HVAC design often lead to equipment over-sizing, causing cycling behaviour of equipment, which result in increased energy use and mechanical wear compared to properly-sized equipment (5).

Especially for heavy, concrete core activated buildings, optimal control is challenging (6), since building, installation and user dynamics span a large frequency range ${ }^{6}$ and since many underlying states are unknown and un-actuated (7).

The effect of persistent degradation faults on energy use is highly depen-

${ }^{6}$ from $5.6 * 10^{-3} \mathrm{~Hz}(0.05 \mathrm{~h})$ for low level control and disturbances, up to $3.9 * 10^{-6} \mathrm{~Hz}$ $(72 \mathrm{~h})$ for heavy building element dynamics 
dent on the type of fault and the fault severity. System performance was reported to be significantly lower when in the presence of faults or when they were induced on purpose. Roth (8) refers to a fault-related "energy waste" in HVAC, lighting and large refrigeration systems between $4 \%$ and $20 \%$. Li (9) investigated the detection and diagnosis of 6 occurring hydronic faults on weather compensated control, optimal start-up and thermal regulation valves on a two-zone school building emulator. In this study, also a questionnaire was sent to 46 maintenance experts of heating systems in France to assess the impact and occurrence of faults in HVAC systems. On average, faults in HVAC installations lead to a 15-40\% increase of yearly energy use $(10 ; 9)$ compared to the fault free situation. These high percentages and wide ranges strengthen our hypothesis that faults should be taken into account in the evaluation of controller performance in office buildings over their lifetime.

The combined effects of uncertainty and control settings for model based controllers have been investigated by Privara (11), Maasoumi (12) and others (13, 14, 15). This research has mainly focused on the impact of model error mismatch due to imperfect parameter estimation and unidentified building dynamics. They documented large swings in control performance, often deteriorating, but sometimes a performance improvement, depending on the degree of model mismatch and the reference used. Also, while some controller formulations had negligible sensitivity even to large model mismatches, others controller algorithms were very sensitive. Castilla (16) made a comparison of thermal comfort predictive control strategies and their possible trade-offs. These observations strengthen our hypothesis that apart from energy use evaluation, also comfort evaluation and supervisory controller types should be taken into account when evaluating control perfor- 
mance in office buildings over their expected lifetime.

Only a limited amount of completed research was found in the area of modelling strategies for common HVAC faults. The development of diagnostic fault models is severely hampered by the scarcity of measured data on the (in-operation) effects of individual faults on HVAC equipment performance. Several studies exist on FDDe performance evaluation in offices, but these fault models and performance evaluations mainly focus on single HVACinstallations (AHU, chiller, boiler). Haves (17) modelled common chiller faults using a first principle model (white box) and an empirical approach (black box model) based on measurements of known (induced) fault states. Braun (18) made a shortlist, frequency table and analysis tool for chiller related faults. Basarkar (19) used the Energy Management run-time Scripting (EMS) functionality in Energyplus (EP) to model and evaluate the energy impact of several VAV box damper failure. Basarkar (19) implemented 5 common AHU faults in Energy Plus using custom EMS schedules. Trcka (20) developed a custom Trnsys type to model common hydronic heating circuit faults. However, faults also occur in building-integrated distribution (e.g. active hydronic controllers) and/or emission systems (e.g. FCU, CCA) and may have a performance impact beyond the system they originate in. In these studies, there was no clear description of what type and severity of persistent faults should be considered when evaluating the economic impact of faults on building-wide HVAC (control) performance in office buildings.

The building-wide impact of (white or colored) sensor- and actuatornoise, additive and multiplicative faults on closed loop HVAC control performance is investigated less often in full building and installation emulations. To the authors knowledge, only Visier (21) investigated faults commonly encountered in six hydraulic heating systems in medium to large size residential 
and non-residential multi-story buildings, for space heating as well as for domestic hot water heating. A heat generation plant with non-condensing gas boilers, with a design temperature around $90{ }^{\circ} \mathrm{C}$ were assumed in this study, including boiler control and sequencing, scheduling of occupancy periods and adaptive control for the temperature setpoint of each secondary loop (RBC) with secondary loop flow control by thermostatic flow-regulation control valves (TRV). An optimum start-stop algorithm for the primary circuit and variable flow temperature control of the temperature difference in the primary loop was assumed. However, this study is not up to par anymore with the current state of technology and state of controllers and this control strategy is not applicable to concrete core conditioned buildings.

In order to actively minimize fault impacts, the occurring fault(s) may be detected, diagnosed and overhauled. Then, if economically feasible and technically possible, the faulty equipment can be overhauled or re-calibrated, or the control algorithm may be reconfigured. A lot of research regarding fault impact and fault detectability in HVAC installations has been performed already and several FDDe (commercially available) products have been developed by academia and industry. However, the FDDe problem in office buildings is complex, since HVAC systems are large in scale, consist of many interacting subsystems, are building- and equipment-dependent and mostly operate under time-varying boundary conditions. From the reviewed literature and BEMS-market investigation (9; 22, 23), it becomes clear that the uptake of FDDe technology is still minimal in BEMS applications, even in high-end buildings with state of the art control systems. Up to this date, it is not common market practice to perform a manual or continuous, regular (yearly or 5-yearly) commissioning in office buildings in order to detect and rectify erroneous control loops or degraded components. 
In this paper, the economic impact of persistent faults are investigated for an hydronic HVAC system in a concrete core activated office building. Note that only persistent faults during the operational lifetime of the sensor and actuator are investigated, not componentfailures (loss of signal or responsivity). As an office building's HVAC system is rarely implemented redundantly, when a component failure occurs, it is required to replace this component to continue normal HVAC operation. This is out of scope of this paper. The focus lies on the (frequently occurring) case in which faulty (but not failing) sensors and actuators with persistent faults are continued to be used for HVAC control.

The evaluated faults include sensor to actuator faults of different severity and combinations of bias, scaling and white noise perturbation of signals. These fault types commonly occur both on input signals (sensors) and output signals (actuators), both in positive and negative sense (increase or decrease of the signal) and at several severity levels, depending on the level of degradation of the HVAC component. Following three generic fault types are investigated:

- BIAS: Additive input faults. These are frequently occurring with a relatively large impact.

- SCALE: Multiplicative errors. These have a lower occurrence, with a potentially large impact.

- NOISE: White noise. These are frequently occurring, with low impact.

An emulator model of an HVAC installation, building and user was developed. For maintainability and reliability, most HVAC components in office buildings are typically decoupled into a production section, distribu- 
tion section and emission section, each equipped with their proprietary low level controllers. They are controlled by external control signals coming from a supervisory controller, mostly residing in a building energy management system. Four different supervisory controller models were developed. Their performance for several fault combinations was evaluated using the resulting simulated energy cost and discomfort costs over a reference year:

- RBC: a rule based, open loop controller based on a heat curve (HC). The weighted historic daily average ambient air temperature is used to determine a supply setpoint.

- OL-MPC: a open loop model predictive controller, pre-calculated using a state space building model both as control model and emulator model.

- CL-MPC: a moving horizon, closed loop model predictive controller, based on a deterministic state space building model.

- R-MPC: a moving horizon robust model predictive controller based on a stochastic state space building model which takes bounded additive sensor and actuator uncertainty into account.

For this evaluation, the control performance of each simulation variant (in fault free and fault-present conditions) is evaluated using two key performance indicators (KPI): energy cost and discomfort cost. These KPIs are summed over a yearly simulation and normalized. Subsequently, these resulting costs for fault free and fault-present situations are merged (per controller) and evaluated over a 10 year horizon using the economic Present Cost (PC) framework. 
The main contributions of this paper revolve around the following three research questions:

- Sensitivity to Faults: Which faults have significant impact on HVAC control KPIs? Is this influence mostly linear, exponential, logarithmic or highly non-linear i.f.o. fault severity? Could this impact be predicted a-priori (for this case study)?

- Fault Robustness: Are there types of (model-based) controllers with which (a-posteriori) fault impact is minimal for this case study? What type of controller is the most robust for a bounded fault severity (for this case study)? Does the fault impact have an effect on the relative ranking of the compared HVAC controllers for this case?

- Fault Detectability: Is there an optimal FDDe detection sensitivity, which limits the KPI degradation for one or more controllers to an allowable, low level? What commissioning frequency and cost would be viable for an optimal economically viable FDDe implementation (for this case study)?

\section{Methodology}

The approach in this paper is to model an office building with an integrated HVAC system, split up in a production, distribution and emission sections, together with model based HVAC controller(s). These are combined and emulated both in FF and fault-present (FP) situations. It would be interesting to validate fault impact, using actual measurement data, should it be available. However, it is theoretically impossible to obtain both reliable fault-free control performance and fault-present performance at the 
same time (with same disturbances) from an office building in use. It would be neither comfortable nor energetically acceptable to induce several severe HVAC faults, which (may) hamper occupant productivity and comfort. Therefore, the simulation based methodology was used here, with the assumption that the emulator behaviour is modelled to sufficient detailed, so it has comparable dynamics to the real reference office building.

As building layout, a mid-section of a multi-story office building with a perimeter landscape layout and a central corridor is represented using three thermal zones: a central corridor in the middle and two office zones at either side. Two generic office zones, in the middle of the office building, each with a different orientation (opposite faade) are considered in the emulator, as the impact of faults on individual zones with non-equal disturbances during the reference year (irradiation, wind, occupation) may be not symmetric. Using this multi-zone emulator, the differences and interaction between the zones can be taken into account too, which could not have been evaluated with a one zone model. The used case study building model, HVAC model and controller models are described in detail in appendix A, for reproducibility purposes.

In the remainder of this section, the focus is placed on the fault emulation, the economic framework, the design of the simulation experiments and weighting (tradeoff) of impact scores.

\subsection{Fault models}

Persistent faults (bias, multiplicative errors and noise) are introduced on sensors and actuators For the fault evaluations, several sensors and actuator models -as described in table 1 - are extended with a parametric bias, scale and noise faults on the signals. Note that faults on flow and supply 
temperature are non-additive in nature and they are highly interactive with temperature faults, as their combined fault effects are reinforced or partially negated.

Similar to the methodology applied by Du (24), input and output errors are added to the output signals from the controllers, or added to emulation data, before sending simulated data to the (supervisory or low level) controllers. As a fault model, following generic structure is used:

$$
x_{h, i, j}=\left(1+m_{h, i, j}\right) * \bar{x}_{h}+a_{h, i, j} * n(\mu, \sigma)+b_{h, i, j}
$$

with $x_{h, i, j}$ the faulty variable value (fault-present), $\bar{x}_{h}$ the true (fault-free) variable value, $a_{h, i, j}$ noise amplitude (the fault amplitude added on random normal distribution), $b_{h, i, j}$ the bias error (additive fault offset), $m_{h, i, j}$ the scaling error (multiplicative fault offset), $n(\mu, \sigma)$ a normally distributed error with mean $\mu$ and variance $\sigma, h$ the (faulty) component index (FC), either $T_{a a} / T_{a s, N} / T_{w s, N}, F l_{w s, N} / T_{o z, N} / F l_{w s, N}$ or $T_{o z, S}, i$ is the fault type (FT), either bias/multiplicative/additive, and $j$ : the fault severity (FS) and sense: either small/mediocre/large and either increase or decrease.

For the univariate and bivariate evaluation of each fault type, a small, medium or large fault severity was selected depending on sensor and actuator type, as given in table 2.1. The values for light and mediocre faults are derived as fractions of the values set for the large fault. These (large) fault values are also used as a starting point for the fault probability assumptions for the LHS analysis.

For the LHS analysis, a normal distribution is assumed for each fault type, with a mean $\mu$ of 0 , and a variance $\sigma^{2}$ of $1 / 4$ (standard deviation $\sigma$ $1 / 2$ ). As the assumed standard deviation, half of the large fault severity was assumed. The corresponding cumulative probability density function 


\begin{tabular}{l|l|l|l|l}
\hline Type of variation & Variant 1 & Variant 2 & Variant 3 & Variant 4 \\
\hline Controller type & RBC & OL-MPC & CL-MPC & R-MPC \\
Sensors (4) & $T_{a a, N \& S}$ & $T_{z o, N \& S}$ & - & - \\
Actuators (5) & $T_{w s, N \& S}$ & $F_{w s, N \& S}$ & $T_{a s, S}$ & - \\
Fault Type (3) & Bias $(A)$ & Scale $($ Gain $M)$ & Noise (ampl. $A N)$ & - \\
Fault severity (3) & Large (L) & Mediocre (M) & Small (S) & - \\
Fault direction $(2)$ & Increase & Decrease & - & - \\
\hline
\end{tabular}

Table 1: Description of the investigated parameter space for the persistent fault impact analysis. Several linear combinations of these different controllers, components (sensors and actuators), fault types, severities and directions are made. Add = Additive, ampl. = amplitude.

\begin{tabular}{ll|lll|lll|lll}
\hline FC & Qty & \multicolumn{3}{|c}{ Add. (B) } & \multicolumn{4}{c}{ Scale (M) } & \multicolumn{3}{c}{ Noise (AN) } \\
& - & $\mathrm{S}$ & $\mathrm{M}$ & $\mathrm{L}$ & $\mathrm{S}$ & $\mathrm{M}$ & $\mathrm{L}$ & $\mathrm{S}$ & $\mathrm{M}$ & $\mathrm{L}$ \\
\hline T-sens. & 3 & $2 / 3$ & $4 / 3$ & 2 & $3.33 \%$ & $6.67 \%$ & $10 \%$ & 0.33 & 0.67 & 1 \\
T-act. & 1 & $2 / 3$ & $4 / 3$ & 2 & $3.33 \%$ & $6.67 \%$ & $10 \%$ & 0.33 & 0.67 & 1 \\
F-act. & 2 & 4.8 & 9.6 & 14.4 & $3.33 \%$ & $6.67 \%$ & $10 \%$ & 0.48 & 0.96 & 1.44 \\
\hline
\end{tabular}

Table 2: Fault severity values used to emulate large faults (L) on different faulty components (FC), for single fault analysis of Temperature (T) and Flow (F) actuators (act.) and sensors (sens.). A small (S) fault is assumed to be at $1 / 3$ of the large (L) value and a mediocre $(\mathrm{M})$ fault severity at $2 / 3$ of the large fault value. Both positive $(+)$ and negative $(-)$ variations are introduced. The large fault parameter values are based on the tabulated process calibration uncertainty in HVAC systems, as reported by Kim (25). Bias and noise are absolute values, while scale is relative $\%$. 
shows that with these assumptions (with sufficiently large population), 50.0

$\%$ of the random LHS-fault realisations will be below the mild level, $81.8 \%$

below the mediocre fault level and $95.4 \%$ below the large fault level.

\subsection{Key performance indicators (KPI)}

For each simulation variant (both fault free and fault-present), the control performance is evaluated using following KPIs, summed over a yearly simulation and normalized to the occupied reference office floor area of the emulation model $\left(24 \mathrm{~m}^{2}\right)$ :

- The energy cost $\left[€ / \mathrm{m}^{2} / y\right]$ is defined as the sum of yearly heating and cooling energy use of the hydronic and the air-sided system $\left[\mathrm{kWh} / \mathrm{m}^{2} / y\right]$, each multiplied by respective system efficiencies [-] and energy price $[€ / k W h]$. For heating, the energy price (for gas) is fixed at 0.05 $€ / k W h$, and for cooling, the energy price for electricity is fixed at $0.20 € / k W h$.

- The discomfort cost $\left[€ / m^{2} / y\right]$ is defined as the sum of yearly underheating and over-heating discomfort, averaged over the two office zones (north and south), multiplied by the average cost of discomfort for the building $\left(0.05 € / K h / m^{2} / y\right)$.

- The maintenance cost $\left[€ / \mathrm{m}^{2} / y\right]$ is estimated based on the type of HVAC installation type,fault occurence and overhaul frequency. For a single fault, a fixed maintenance fee of $5 / \mathrm{m}^{2}$ is assumed per intervention. For an LHS fault pattern, a maintenance budget of $25 / \mathrm{m}^{2}$ is foreseen for detection and replacement of the faulty component(s). This cost is seen as an investment cost and is discounted completely to year zero of the economic evaluation period. 
The average estimated productivity decrease and estimated average occupant wage and occupation density are combined to obtain a single weighting parameter that quantifies the economic impact of discomfort. When assuming a linear productivity decrease of $3 \%$ for a weighted discomfort of $2 K h(26)$, an area weighted gross personnel wage cost of $6.000 € / m^{2} / y$, and $1800\left[h_{\text {working }} / y\right]$ working hours per year, the scaling value of this discomfort weighting parameter becomes $0.05 / \mathrm{Kh} / \mathrm{m}^{2}$. With this discomfort cost weighting parameter, a simulated or calculated weighted sum of yearly thermal discomfort $[K h / y / z o n e]$ can be directly converted to a comfort-related cost. The limit of $100 \mathrm{Kh} / \mathrm{y}$ acceptable overheating and undercooling, corresponds to a maximal acceptable discomfort cost of $5 / \mathrm{m}^{2} / y$ one-sided evaluation of office buildings (or $10 / \mathrm{m}^{2} / y$ when evaluating both heating and cooling).

\subsection{Unified evaluation criterion}

For evaluating and comparing costs over multiple years, the present cost (PC) method is used, where yearly costs (e.g. energy cost, discomfort cost) are depreciated over the life time horizon of the controller and discounted to the year 0 . In addition to this yearly cost, there are several other related costs that are case and controller dependent, but only occur once over the lifetime of the controller, such as replacement costs, investment costs and (re-)commissioning costs. To take these into account, formula 2.3 is used:

\footnotetext{
${ }^{7}$ The average annual office personnel cost $[€ / y]$ weighted to floor area $\left(m^{2}\right)$ for office buildings is estimated between 2.000 and $12.000 € / \mathrm{m}^{2} / y$, assuming an average occupancy of 0.1 person $/ \mathrm{m}^{2}$ and an average gross yearly wage of 20.000 to $120.000 €(27)$
} 


$$
\begin{array}{rr}
C_{P C_{y=0}, C T}= & w_{P C, C} * C_{C, C T, \star} \\
+\sum_{y=0}^{t_{\text {eval }}=10-1} & \frac{C_{E, y, C T, \star} *\left(1+i_{E}\right)^{y}}{\left(1+i_{I}\right)^{y}} \\
& +\frac{C_{D, y, C T, \star} *\left(1+i_{W}\right)^{y}}{\left(1+i_{I}\right)^{y}}
\end{array}
$$

with $C_{x}$ the associated costs, which can be either total present cost (PC), capital investment cost (C), energy cost (E) or Discomfort cost (D); CT the supervisory controller type: either RBC, OL-MP, CL-MPC or R-MPC; $y$ the simulated yearly KPI performance; $i$ the yearly inflation rate for energy and wages and $\star$ denoting the corrected value, including effects of commissioning. This equation can be rewritten as a linear combination of three economydependent weights, that can be pre-calculated independent of the associated base costs, as shown in equation 3

$$
\begin{aligned}
C_{P C, C T}= & w_{P C, \text { investments }} * C_{\text {investment }, C T} \\
& +w_{P C, e+i} * C_{\text {Energy }, y, C T, *} \\
& +w_{P C, w+i} * C_{D i s c, y, C T, \star}
\end{aligned}
$$

with $w$ the fixed weight factors, $e+i$ the effect of energy and inflation, and $w+i$ the factor related to wages and inflation.

The yearly inflation rate $i_{\text {infl }}$ assumed in this paper is fixed at $2.2 \%$ (28) based on the 5 years forward 5 years ahead estimates from the European central bank (28). The expected yearly change in energy cost $i_{\text {energy }}$ is also fixed at $2.2 \%$, as the energy cost is a primary driver of the index and 
therefore is closely related to the inflation rate. The expected yearly wage cost change $i_{\text {wages }}$ at $3.1 \%$ based on figures published by Hay (29).

A sensitivity study on these parameters (between 0 and $4 \%$ for each parameter) shows that the relative tradeoff ratio of energy cost against maintenance and discomfort cost barely changes when inflation rate changes (influence on the ratio over the investigated range stays below $2 \%$, ceteris paribus), but relative shifts of energy cost versus wage depreciation does have a major influence on the cost scaling $(-16 \%$ to $+20 \%$ change of tradeoff ratio). A steeper energy price increase rate relative to wage increase, decreases the relative cost of discomfort and maintenance, which favours more energy efficient controllers but disfavours regular (more costly) maintenance. On the other hand, a reduction of assumed energy price raise (relative to wage increase) favours regular maintenance and controllers which aim for good comfort.

The controller evaluation horizon $t_{\text {eval,c }}$ lifetime is fixed at 10 years. The cost calculation depreciates the related costs to year 0 as presented in equation 2.3. Based on these assumptions, the investment cost weight $w_{P C \text {,investments }}$ is 1.00 , the energy and inflation related weight $w_{P C, e+i}$ is 10.41 and the wages and inflation related weight $w_{P C, w+i}$ is 10.00 .

$$
C_{k, \text { mean }}=\frac{\left(w_{F F, k} * C_{k, P C, F F}\right)+\sum_{F C=1}^{n_{F C}} \sum_{F T=1}^{n_{F T}} \sum_{F S=1}^{n_{F S}}\left(w_{F P_{F T, F S}} * C_{*, P C, F C, F P_{F T, F S}}^{\star}\right)}{\left(w_{F F, k} * n_{F F}+w_{F P_{F T, F S}} * n_{F C} * n_{F T} * n_{F S}\right)}
$$

with index $k$ denoting the type of KPI: energy or discomfort cost, index $C T$ denoting the supervisory controller type, index $F C$ the faulty component (FC) type, index FT the fault type and FS the fault severity. Since some large faults, or faults with large comfort impact might be easily detected 


$$
\begin{aligned}
& C_{t, F P, \text { yearly }, C T, F C, F T, F S}^{\star}= \\
& \begin{cases}C_{t, F P, C T, F C, F T, F S} & \text { if } C_{D i s c, F P, C T, F C, F T, F S} \leq C_{\text {Disc,limit }} \\
\beta * C_{t, F F, C T}+(1-\beta) * C_{t, F P, C T, F C, F T, F S} & \\
+1 / 2 * w_{P C, w+i} * C_{c o m m, F C} & \text { if } C_{D i s c, C T, F P, F C, F T, F S}>C_{\text {Disc,limit }}\end{cases}
\end{aligned}
$$

For the LHC sampling tests, the control performance results can be summarized similarly. Also here, when the associated discomfort cost is unlikely high (above a discomfort detection limit $C_{d i s c, l i m i t}$, it is assumed that facility management detects the fault prematurely, and shifts the control towards fault free (or fault-reduced control) situation again. This will come at a cost $C_{\text {comm }, L H S}$, related to fault detection, and equipment overhaul. Also here (similar to equation 5) a suitable estimate of commissioning frequency $\beta$ and cost per intervention is required. For this case study, the $\beta$ factor is chosen to be $50 \%$ (which corresponds to a commissioning frequency once every 5 years), and the added commissioning cost $C_{c o m m, h}$ is fixed at $5 € / m^{2} / y$ for single faults and $25 € / \mathrm{m}^{2} / y$ for LHS faults according to: 
$C_{L H S, C T}= \begin{cases}\sum_{l=1}^{n_{L H S, C T}}\left(C_{t, F P, l, C T}\right) / n_{L H S} & \text { if } C_{l, \text { disc }} \leq C_{\text {disc,limit }} \\ \sum_{l=1}^{n_{L H, C T}}\left(\beta * C_{t, F F, C T}+C_{c o m m, L H S}\right. & \left.+(1-\beta) * C_{t, L H S, C T}\right) / n_{L H S} \\ & \text { if } C_{l, \text { disc }}>C_{\text {disc,limit }}\end{cases}$

with $C_{t, F F, C T}$ the fault-free performance cost relating $t$ (energy or discomfort) for controller type $\mathrm{CT}$ and $C_{t, L H S, l, C T}$ the KPI value relating $t$ (energy or discomfort) corresponding with the LHS-sample with index $l$, and with $l$ the index for the LHS realization (simulation), For this paper, $n_{L H S, C T}$ was fixed at 20 .

\subsection{Experiment setup}

Using this emulator model and building control models, a large subset of HVAC types, controller models and fault variations were emulated. As simulation settings, the simulation duration was chosen at one year, with a low level control timestep of 12 minutes, and a supervisory control timestep of $1 \mathrm{hr}$.

For a single fault occurrence in combination with the RBC and OLMPC, every linear combination (one faulty component and one fault type at a time, 1512 variants) is evaluated. For the other controller variants, this full analysis is not performed, due to the long calculation times for these simulations and due to the similar profiles obtained for RBC and OL-MPC.

For bi-variate fault occurrence (two fault types persisting simultaneously on different sensors or actuators), all combinations of a mild fault and any single other fault severity was evaluated, but only in combination with controller type RBC (2268 combinations). 
For a combined fault evaluation, simultaneous stochastic fault occurrences (normally distributed around fault-free condition) on all sensors and actuators are investigated. On this vast search space, a Latin hypercube sampling (LHS) with an assumed normally distributed fault occurrence for each fault severity was performed. For each supervisory controller, 20 cases are simulated.

\section{Simulation Results}

\subsection{Fault free analysis}

The developed emulator (described in appendix I) is used to simulate the FF and FP performance of the different case studies and controllers. For each combination of CT and (set of) FT, yearly simulations are executed. An exemplar resulting weekly zone temperature profile in fault-free conditions are shown in figures 1 and 2 , for the four controller types, together with their hydronic and aereolic set points and energy use. From these simulation runs, the economical KPIs for yearly energy and yearly discomfort cost $C_{t, F P, C T, F C, F T, F S}\left[\Theta / \mathrm{m}^{2} / y\right]$ are computed for each evaluated univariate, bivariate or LHS fault combination: One for yearly discomfort cost and one for yearly energy cost.

Fault-free control performance is shown in figures 1 and 2, During spring season (figure 1), the R-MPC and CL-MPC maintain a slightly lower temperature than the other two controllers, but each controller is able to maintain comfortable zone conditions. The RBC has a slightly higher average operative temperature, both because of a higher initial zone temperature and from heating slightly too much. CL-MPC and R-MPC are able to maintain the zone temperature in the thermal comfort band in a more energy-efficient 
way, by utilizing the air-based installations more, while OL-MPC mostly provides cooling through the water-based installation. In winter season and in fault-free conditions (figure 2), the more conservative R-MPC steers the operative zone temperatures to the center of the comfort band, mostly using the air sided temperature control, resulting in a heating energy cost similar to the reference control (RBC), which is designed with a fixed heating curve air supply temperature. However, the R-MPC uses a quite aggressive changeover strategy. This can be explained as the R-MPC minimizes the sum of energy use and discomfort attained under worst case additive uncertainty, while this additive fault is not present here.

Comfort and energy performance on a yearly basis can be evaluated visually based on the yearly temperature duration curves and yearly load duration curves. These are shown in figures 3 and 4 for all controller types in FF conditions. From the production load duration curve (figure 4), it can be seen that both CL-MPC and R-MPC utilize a full load capacity more frequently than the other controllers, but do not operate as often in part load. The temperature profiles are comparable, with a slight increase of under-heating discomfort attained by the R-MPC, and a slight increase of under-cooling by OL-MPC and (conservative) min-max R-MPC. On the other hand, in fault-free conditions, the temperature duration and load profiles for OL-MPC and RBC are similar, with only some variations within the thermal comfort band.

The weighted (fault-free) PC performance of all controllers are all summarized in table 3. This table shows that under fault-free conditions, the OL-MPC has the best performance of all evaluated supervisory controllers.

Especially the discomfort cost is minimal for this controller, but also the energy use cost is relatively low (though a bit higher than the CL-MPC). 


\begin{tabular}{l|l|lll}
\hline Controller type & Present cost & Energy Cost & Discomfort cost & Maint. cost \\
$\mathrm{CT}$ & $C_{P C, C T}$ & $C_{E, L H S, C T}^{\star}$ & $C_{D, L H S, C T}^{\star}$ & $C_{M, F P, y, C T}$ \\
- & {$\left[€ / m^{2} / 10 y\right]$} & {$\left[€ / m^{2} / y\right]$} & {$\left[€ / m^{2} / y\right]$} & {$\left[€ / m^{2} / y\right]$} \\
\hline $\mathrm{RBC}$ & 60 & 2.69 & 3.18 & 0 \\
OLMPC & 52.5 & 2.45 & 2.68 & 0 \\
CLMPC & 81 & 3.41 & 4.51 & 0 \\
RMPC & 50 & 3.2 & 1.73 & 0 \\
\hline
\end{tabular}

Table 3: Present Cost for each simulated controller in fault-free (FF) conditions

\subsection{HVAC Performance under persistent Faults}

In the subsequent graphs 5 and 6 , the uni-variate and full search space (LHS) fault simulation results are summarized in a scatter plot of HVAC energy cost versus discomfort cost, based on $[K h / y]$. For each controller, also the fault-free KPI performance and the average PPD [\%] in fault-free and fault-present are given in comparison in table 4. Graph 5 and 6 show the impact of a single fault, in combination with the RBC and OL-MPC respectively, as a function of type of fault and ordered by fault severity for each faulty component. On these graphs, it can be seen that single faults have a wide range of impact on energy use and comfort, which both depend on the faulty component and the type of fault. Especially comfort is negatively affected when a single fault occurs. With RBC, the zone temperature sensors $T_{o, N}$ and $T_{o, S}$ are the most critical components (lowest robustness). The sensitivity to $T_{w s}$ and $T_{a a}$ faults is minimal. On the other hand, the OL-MPC is quite sensitive to faults on $T_{w s}$ and $T_{a a}$, especially with bias errors.

For most univariate faults, the impact on comfort is negatively correlated to the impact on energy use. However, note the scale difference in the graphs: 


\begin{tabular}{c|cc}
\hline Simulation runs & WUH & PPD \\
- & mean, stdev $[K h]$ & mean, stdev [\%] \\
\hline RBC, FF (1) & $79.4,0$ & $10.76,0$ \\
RBC, LHS (20) & $198.2,675.2$ & $10.76,2.03$ \\
OL-MPC, FF (1) & $67.1,0$ & $8.91,0$ \\
OL-MPC, LHS (20) & $273.3,839.0$ & $8.28,0.35$ \\
CL-MPC, FF (1) & $52.6,0$ & $8.23,0$ \\
CL-MPC, LHS (20) & $111.0,497.3$ & $9.06,1.66$ \\
R-MPC, FF (1) & $43.2,0$ & $7.33,0$ \\
R-MPC, LHS (20) & $320.9,38.5$ & $7.31,1.05$ \\
\hline
\end{tabular}

Table 4: Comparison between average and standard deviation of the thermal comfort performance of several fault scenarios and controller types, expressed in two discomfort performance indiches: Weighted undercooling/underheating hours (WUH) and Percentage of people dissatisfied (PPD) . For PPD, a clothing factor of 1 clo was assumed, with a metabolic rate of 1.2 met and a relative air velocity of $1 \mathrm{~m} / \mathrm{s}$. Note that average increase of yearly averaged PPD of fault-present versus fault-free reference is often small while the incurred discomfort in $[\mathrm{Kh}]$ is relatively large. The variance of WUH metric is larger than the variance in PPD. Note that only thermal comfort is evaluated with the WUH methodology, while PPD evaluates hygrothermal comfort. Therefore, the $[K h]$-index is a more appropriate and sensitive thermal discomfort KPI for our purpose. 
the absolute impact of a single fault on cost comfort is typically 10 times higher than the effect on energy use. Therefore, the combined impact of a discomfort cost increase and a (slight) energy cost decrease, typically results in a net cost increase.

Graph 7 summarizes the impact of simultaneous (normally distributed) faults on all sensors, on control performance of the four evaluated controllers. The squares and dotted line (bottom side of the graph) depict the theoretical minimum energy use with limited but perfectly controlled HVAC emission capacity, determined using a simple set point controlled low level controller. As most points have shifted to the right on this graph compared to the reference, instead of upwards, it can be concluded that multiple simultaneous (LHS) faults have an especially detrimental impact on discomfort cost (up to $+1200 \%$ for the evaluated severities) while they have a much smaller, but non-negligible impact on energy cost (up to $+350 \%$ for the evaluated severities). Note that this graph is not scaled for the macro-economic effects over the lifetime of the controller. As the weighting factors for each parameter is slightly different, a slight tilt in the equi-cost lines will occur, when these effects are taken into account.

Following the economic framework and weighting methodology described in the previous chapter, these KPI's are merged, economically weighted and summarized in table 5 . The contributing reference cost and simulation output datapoints are presented in table 3 .

The possibility of resetting the fault impact towards the fault-free performance when a high discomfort is detected (e.g. above $100 \mathrm{Kh} / \mathrm{y}$, corresponding to $10 € / \mathrm{m}^{2} / y$ ), was also taken into account. For the LHS fault detection and overhaul, a commissioning cost of $25 € / \mathrm{m}^{2} /$ intervention every two years was assumed (a weighted sum of $20 \%$ of average LHS performance 


\begin{tabular}{l|l|lll}
\hline $\begin{array}{l}\text { Controller type } \\
\text { CT }\end{array}$ & Present cost & Energy cost & Disc. cost & Maint. cost \\
- & $C_{P C, C T}$ & $C_{E, L H S, C T}^{\star}$ & $C_{D, L H S, C T}^{\star}$ & $C_{\text {Maint }, F P, y, C T}$ \\
{$\left[€ m^{2} / 10 y\right]$} & {$\left[€ / m^{2} / y\right]$} & {$\left[€ / m^{2} / y\right]$} & {$\left[€ / m^{2} / y\right]$} \\
\hline RBC & 109.7 & 3.83 & 6.24 & 0.63 \\
OLMPC & 115.3 & 3.23 & 6.72 & 1.25 \\
CLMPC & 89.3 & 3.79 & 4.06 & 0.88 \\
RMPC & 110.6 & 3.16 & 6.85 & 0.75 \\
\hline
\end{tabular}

Table 5: Present Cost for each simulated controller under the presence of LHS faults, taking into account commissioning of faulty components in high-discomfort cases at a fixed commissioning frequency (Corrective weight: $20 \%$, commissioning cost: 25 $€ / m^{2} /$ intervention).

and $80 \%$ of the FF performance is used). This corresponds to a bi-yearly overhaul rate. These last two parameters are linked (commissioning cost and overhaul performance), as an increase of the number of interventions will also increase the yearly maintenance cost.

An optimum cost trade-off was determined for each controller as shown visually in figures 9. The results are presented in table 6. This approach, based on formula 6 is broken down into constituents at different values of $\beta$ for the RBC strategy in figure 8 . The energy cost and discomfort decline with increased commissioning frequency $\beta$ (increased maintenance periodicity), but the associated maintenance costs also rise. Therefore, an optimum commissioning frequency or optimal commissioning time window exists for each controller type.

Finally, figure 10 summarizes all LHS simulation PCs for all controllers in one graph, both for the reference case, the fault present case without commissioning, a case with commissioning at regular, fixed interval and at an optimal maintenance periodicity (see table 6 for more details). 


\begin{tabular}{l|l|ll}
\hline $\begin{array}{l}\text { Controller type } \\
\text { CT } C_{P C, C T, \text { opt }}\end{array}$ & $\begin{array}{l}\text { Tuned PC } \\
\beta * 10 y\end{array}$ & $\begin{array}{l}\text { Optimal comm. period } \\
C_{\text {Maint }, \text { F P,yearly,CT }}\end{array}$ & Maint. cost \\
- & {$\left[€ / m^{2} / 10 y\right]$} & year & {$\left[€ / m^{2} / y\right]$} \\
\hline RBC & 107.9 & 3.85 & 1 \\
OLMPC & 113 & 4.96 & 2 \\
CLMPC & 79.1 & 3.47 & 0.6 \\
RMPC & 92.1 & 2.94 & 1.5 \\
\hline
\end{tabular}

Table 6: Minimal present cost (PC) for each simulated controller under the presence of LHS faults, with optimized commissioning period (frequency) which minimizes the PC (with corresponding yearly commissioning cost equal to $\left(25 /\right.$ intervention) $\left.€ / \mathrm{m}^{2} / y\right)$.

\section{Discussion}

From simulation results, summarized in marginal fault impact graphs (figures 5 and 6) and the economical KPI tables (tables 3,5 and 6) shown in the previous section, several conclusions can be drawn. In this paper, the focus lies on three research questions related to sensitivity, robustness and detectability, which guide the analysis.

\subsection{Sensitivity}

Regarding the fault sensitivity of the different $\mathrm{MBC}$, the primary research question was, if it is needed to take the fault impact into account during $\mathrm{MBC}$ evaluation? It was expected that $\mathrm{RBC}$ would be least robust to faults and would be especially fault-sensitive to temperature sensor and actuator bias faults.

The impact of single faults to one controller are mostly linearly correlated, as seen in figure 5 and 6 , but for some faulty components, there is a quadratic (e.g. zone air temperature sensors), or higher order polynomial (e.g. supply water temperature sensor, flow actuator). Especially bias errors 
on zone temperature and ambient temperature faults have a large impact. For noise errors, there is no influence of fault direction, which was expected, as the fault is a stochastic, normally distributed noise with mean 0 was assumed.

The impact of a single fault on energy use and discomfort are mostly negatively correlated, as a fault that causes an increase on one KPI, often reduces another one. However, some faults cause an increase of both KPIs, for example noise errors on a zone temperature sensor $\left(T_{o} S\right)$. For this case study, the impact on discomfort is a lot larger for most CT and FT, a (large) increase of discomfort cost invariably causes an increase of the PC, regardless of the potential positive (decreasing) effect on energy cost. Therefore, the exact FI is very case/controller dependent and highly sensitive to the assumptions made, which makes this impact is very hard to predict accurately a priori.

Based on the single fault impact results, it can be inferred that there is a large sensitivity to bias faults of the zone temperature sensors. Simultaneous faults, especially increase of HVAC supply flow rate, seem to aggravate these effects.

The absolute fault impact is the largest for the OL-MPC and R-MPC (+67.0 and $52.8 € / \mathrm{m}^{2} /$ lifetime respectively) and much smaller for the other two evaluated controllers $\left(+15.1\right.$ and $+48.7 € / m^{2} /$ lifetime respectively). This is to be explained as the closed loop controllers (CL-MPC and RBC) can make corrective actions when a fault causes discomfort, while the OLMPC has no means to react to these faults on a supervisory level, but undergoes the associated comfort penalties passively. However, the buffering effect of the heavy capacity building, and the fact that a supply temperature is used as a signal variable and not a heat flux, does negate part of the com- 
fort cost at the cost of increased energy usage. Therefore, the energy use of the closed loop controllers increases substantially more in the FP situation than energy use of the OL-MPC (which increases slightly). Moreover, the production capacity is used more often at full load conditions, as seen in figure 1. This corrective action, leads to a reduction of the discomfort impact, but also leads to an increase of the energy cost.

The relative impact of combined faults on KPI's is determined based on LHS and then weighted for economical factors and commissioning as summarized in table 5. Without commissioning, the average combined fault impact is substantial for most controllers. It corresponds to an increase of the PC of $113 \%$ (for RBC), +160\% (for OL-MPC) and even up to $197 \%$ (for R-MBC) compared to FF performance. The CL-MPC is able to provide satisfactory performance despite the presence of faults, even without regular commissioning. Un, it outperforms the other controllers and only has a +17 $\%$ increase against reference, fault-free performance. Note however, that a perfect prediction of weather disturbances and occupancy are assumed. This controller is by far the most fault tolerant of the investigated controller types and fault ranges.

\subsection{Robustness}

The mean impact on energy, comfort and maintenance cost, both in fault free and fault present situation is shown graphically in 10 and summarized in table 5 for each controller and scenario. Figure 7 shows the point cloud of the raw data and the summarized total PC for each controller. It can be seen that the relative robustness is indeed better for CL-MPC and RBC than for R-MPC and OL-MPC, as these point clouds are less spread out, especially in the energy dimension. 
When neglecting the comfort related costs, in the presence of persistent faults, the OL-MPC would be the optimal choice, as it has the lowest energy cost (with discomfort infringements) for this case. When only discomfort cost is considered, the R-MPC shows the best performance. However, in the presence of faults and when including both energy use and discomfort cost are weighted with realistic costs, the OL-MPC performance deteriorates drastically with a change of associated PC. At an overhaul reaction rate of $50 \%$ (every five years) and a (high) cost of 25 Eper intervention for this case, the CL-MPC outperforms the other investigated options, with a total PC of $96.2 € / m^{2} /$ lifetime attributed to the occurring faults, followed by the R-MPC with an PC of $102.8 € / \mathrm{m}^{2} /$ lifetime.

The simulation results show that CL-MPC has the lowest overall fault sensitivity. While the R-MPC does show a significant increase of discomfort cost due to faults, no significant increase in energy use was detected in the fault present case. Therefore, this controller implementation has a good robustness to energy cost. The RBC has a moderate overall fault sensitivity.

When regular commissioning is taken into account, the relative impact of faults is lowered to $+80 \%$ (for RBC), $+119 \%$ (for OL-MPC) and +106 $\%$ (for R-MPC). Again, the CL-MPC outperforms the other controllers under these settings and commissioning interval, with a relative impact on PC limited to $+14 \%$. This wide spread supports our conclusion that fault sensitivity should be taken into account when evaluating controller performance.

\subsection{Detectability}

A fault detection system (to identify and correct faulty behaviour) and suitable commissioning schedule is critical to obtain a good control performance. 
Since the impact of univariate medium and large bias faults on the sensors $T_{o a, S}$ and $T_{o a, N}$ are large for all investigated control algorithms (400

to $800 \%$ ), striving towards increased accuracy or bias fault detection on these sensors has a large economic value. Sensor bias reduction can be realized by adding redundancy on the sensor side or by executing regular sensor calibration. Improved sensor precision, which lowers sensor noise, can be obtained by averaging multiple subsequent measurements. Alternatively, user feedback could be taken into account, since when appropriate communication channels are set up, the employees can quickly detect and rapport uncomfortable zones.

With realistic maintenance costs assumptions, the economic benefits generated by a regular overhaul are shown to be maximized (cost minimized) when performed every 3 to 5 years (see table 6), as they lead to minimal PC under the economical assumptions and based on the simulation results in this case study.

With a high commissioning frequency (every 3 years), both RBC and R-MPC perform almost equally well economically in this case study. At a commissioning rate of once per 5 years, the CL-MPC had the lowest PC of all investigated controllers. The total cost, calculated as the (weighted) sum of energy use, discomfort and maintenance costs for this case study, in the presence of persistent HVAC-related faults and with regular commissioning was the lowest $\left(79.1 € / \mathrm{m}^{2} /\right.$ lifetime $)$ for the CL-MPC, as seen in table 6 .

\section{Conclusion}

This paper illustrates a economical performance evaluation methodology to examine the performance of HVAC-controllers under the influence of 
persistent degradation faults, in contrast to the often superior fault-free performance. This is demonstrated using an emulated case study of a thermally heavy office building equipped with concrete core activation and controlled by one of four different supervisory control algorithms.

The impact of single degradation faults on temperature and flow sensors and actuators is found to be non-negligible and non-linear. Especially bias errors on the zone temperature sensors have a large impact on supervisory control comfort performance. Also faults (bias and noise) present on the water supply temperature control temperature signal severely deteriorate the control performance.

The combined impact of simultaneous faults varies widely for the different controller types and fault combination instances $(-14 \%$ to $+1100 \%$ against the reference for the evaluated controller). The resulting average present costs for different supervisory controllers under the influence of simultaneous, stochastic distributed faults, are consistently higher than their fault free reference performance $(+17 \%$ to $+200 \%)$.

A large variation in controller robustness against persistent HVAC faults was observed. Fault sensitivity caused a change in the relative economic order of the supervisory controllers for this case study. While in the fault free situation, the R-MPC was superior and CL-MPC was the least favourable, the CL-MPC and RBC proved to be economically superior when LHS-faults were considered and frequent commissioning was performed. However, the R-MPC excelled at low (or no) commissioning rates. The OL-MPC proved to be very sensitive to common HVAC-faults. For this case and fault assumptions, the CL-MPC was the most robust controller. With optimal commissioning frequencies, a relative increase between $+10 \%$ and $+198 \%$ was observed in total associated present costs. 
671

Commissioning (investments in recalibration and tuning of smart/accurate sensors) is shown to be be critical and profitable, as it can reduce the control related costs significantly by overhauling faulty equipment and thereby resetting the control performance to the reference performance. The optimal commissioning frequency for faulty control-related equipment is calculated. It ranges between 3 to 5 years for most controllers. However, this economical commissioning frequency for multi-sensor overhaul is very dependent on the emulated case and on economic and controller parameters.

As these conclusions are only supported by an emulation study for this type of building (Heavy building with CCA) and under fixed assumptions, following topics are recommended for further research: Extending this methodology to other types of buildings (e.g. lower thermal mass or different insulation thickness), other types of HVAC systems (e.g. fan coil units, all air systems, etc) and broader fault types (e.g. coloured noise).

\section{Acknowledgments}

The author wishes to thank the KULeuven Mechanical engineering technology cluster for their financial support, which made this research possible.

\section{Nomenclature}

Following abbreviations have been used in this manuscript:

\begin{tabular}{l|l} 
Acronym & Description \\
\hline$a d d:$ & Additive (or offset) fault \\
$A H U:$ & Air handling unit \\
$A N:$ & Amplitude of normally distributed noise
\end{tabular}




\begin{tabular}{l|l}
$B:$ & Bias, see add \\
$B E M S:$ & Building energy management system \\
$B A S:$ & Building automation system \\
$C A V:$ & Constant air volume (ventilation) \\
$C C A:$ & Concrete core activation \\
$C L C:$ & Control life cycle (evaluation period) \\
$C L-M P C:$ & Closed loop MPC \\
$C T:$ & Controller type \\
$E M(C) S:$ & Energy management and control system \\
$E S C O:$ & Energy servicing company \\
$F C:$ & Faulty component \\
$F D D e:$ & Fault detection, diagnosis and evaluation \\
$F F:$ & Fault free \\
$F I:$ & Fault impact \\
$F P:$ & Fault present (situation) \\
$r F P:$ & Random fault propagation
\end{tabular}

672 Following symbols have been used in this manuscript:

673

\begin{tabular}{l|l} 
Symbol & Description \\
\hline$C$ & Financial impact $($ Cost $)\left[€\right.$ or $\left.€ / \mathrm{m}^{2} / y\right]$ \\
Taa & Ambient air temperature $\left[{ }^{\circ} \mathrm{C}\right]$ \\
Tas & Suppy air temperature $\left[{ }^{\circ} \mathrm{C}\right]$ \\
Tws & Suppy water temperature $\left[{ }^{\circ} \mathrm{C}\right]$
\end{tabular}




\begin{tabular}{|c|c|}
\hline Toz & Operative zone temperature $\left[{ }^{\circ} \mathrm{C}\right]$ \\
\hline$F / F l$ & Flow rate $[\mathrm{kg} / \mathrm{h}]$ \\
\hline$N / S$ & (subscript) Reference to the North or South zone \\
\hline$P C$ & (subscript) Reference to Present cost, discounted to reference year 0 \\
\hline$h$ & (subscript) Faulty component (FC) index \\
\hline$i$ & (subscript) Fault type (FT) index \\
\hline$j$ & (subscript) Fault severity (FS) index \\
\hline$\mu$ & Mean \\
\hline$\sigma^{2}$ & Variance \\
\hline $\mathrm{AN}$ & Amplitude of noise error \\
\hline A & Offset of bias error \\
\hline $\mathrm{M}$ & Gain of scale error \\
\hline $\mathrm{S}$ & Small FS \\
\hline $\mathrm{M}$ & Mediocre FS \\
\hline $\mathrm{L}$ & Large FS \\
\hline$E / D / M$ & (subscript) Reference to either Energy, Discomfort or Maintenance \\
\hline$y$ & (subscript) year \\
\hline$C T$ & (subscript) controller type \\
\hline$i$ & Inflation rate \\
\hline$E / W / I$ & (subscript) referring to energy cost, wage indexation and price inflation \\
\hline$w$ & Weight parameter \\
\hline$F F$ & (subscript) Fault Free \\
\hline$F P$ & (subscript) Fault present \\
\hline$n$ & Quantity of evaluated fault realisations \\
\hline$t$ & Index of multivariate fault realisation \\
\hline$\beta$ & Commissioning frequency \\
\hline
\end{tabular}




\begin{tabular}{l|l}
$T_{v s / r}$ & Air supply or retour temperature $\left[{ }^{\circ} \mathrm{C}\right]$ \\
$T_{c}$ & Concrete Core temperature $\left[{ }^{\circ} \mathrm{C}\right]$ \\
$T_{s}$ & Surface temperature $\left[{ }^{\circ} \mathrm{C}\right]$ \\
$T_{i a}$ & Indoor air temperature $\left[{ }^{\circ} \mathrm{C}\right]$ \\
$T_{w r}$ & Water retour temperature $\left[{ }^{\circ} \mathrm{C}\right]$ \\
$m_{w}$ & Water supply mass flow rate $[\mathrm{kg} / \mathrm{h}]$ \\
$m_{w 2}$ & Bypass flow rate $[\mathrm{kg} / \mathrm{h}]$ \\
$m_{v s / r s}$ & Air supply mass flow rate $[\mathrm{kg} / \mathrm{h}]$ \\
$T_{h / c, p r o d}$ & Plant water supply temperature production for heating and cooling $\left[{ }^{\circ} \mathrm{C}\right]$
\end{tabular}

\section{Bibliography}

[1] S. Katipamula, M. Brambley, Review Article: Methods for Fault Detection, Diagnostics, and Prognostics for Building SystemsA Review, Part II, HVAC\&R Research 11 (2) (2005) 169-187. doi:10.1080/10789669. 2005.10391133.

[2] N. Djuric, Real-time supervision of building HVAC system performance, Ph.D. thesis, Norwegian Univrsity of Science and technology (2007).

[3] G. Huang, F. Jordán, Model-based robust temperature control for VAV air-conditioning system, HVAC and R Research (3) 432-445. doi: $10.1080 / 10789669.2012 .649879$

[4] S. Katipamula, M. Brambley, Review Article: Methods for Fault De- 
tection, Diagnostics, and Prognostics for Building SystemsA Review, Part I, HVAC\&R Research (1) 3-25. doi:10.1080/10789669.2005. 10391133 .

[5] S. Wu, K. Neale, M. Williamson, M. Hornby, Research opportunities in maintenance of office building services systems, Journal of Quality in Maintenance Engineering 16 (1) (2010) 23-33.

[6] W. Gntensperger, M. Gwerder, A. Haas, B. Lehmann, F. Renggli, J. Tdtli, Control of concrete core conditioning systems , Proceedings of the 8th REHVA World Congress for Building Technologies CLIMA 2005.

[7] M. Sourbron, Dynamic thermal behaviour of buildings with concrete core activation, Ph.D. thesis.

[8] K. Roth, D. Westphalen, M. Feng, The Energy Impact of Faults in U.S. Commercial Buildings, International Refrigeration and Air Conditioning Conference. Paper 665.

[9] S. Li, A Model-Based Fault Detection and Diagnostic Methodology for Secondary HVAC Systems, Drexel University.

[10] K. J.Y., E. Pierce, Sensor errors and their effecto $n$ building energy consumption, ASHRAE 25.

[11] S. Prívara, Building modeling and identification for predictive control, Ph.D. thesis, Czech Technical university in prague.

[12] M. Maasoumy, Controlling Energy-Efficient Buildings in the Context of the Smart Grid : A Cyber-Physical System Approach Smart Build- 
713

ings in the Smart Grid, Ph.D. thesis, University of California, Berkeley (2013).

[13] M. Trčka, J. L. M. Hensen, Overview of HVAC system simulation, Automation in Construction (2) 93-99. doi:10.1016/j.autcon.2009. 11.019 .

[14] D. Gyalistras, Final report Use of Weather and Occupancy Forecasts for Optimal Building Climate Control (OptiControl) 1. Management Summary (July).

[15] S. C. Bengea, A. D. Kelman, F. Borrelli, R. Taylor, Model Predictive Control for Mid-Size Commercial Building HVAC : Implementation , Results and Energy Savings, HVAC\&R Research 20 (2014) 979-986.

[16] M. Castilla, J. Álvarez, M. Berenguel, F. Rodríguez, J. Guzmán, M. Pérez, A comparison of thermal comfort predictive control strategies, Energy and Buildings (10) 2737-2746. doi:10.1016/j.enbuild. 2011.06 .030

[17] T. S. Haves P., J. Wright, Condition monitoring in HVAC subsystems using first principles models, ASHRAE Transactions 102.

[18] J. E. Braun, R. W. H. Laboratories, Automated fault detection and diagnostics for vapor compression cooling equipment, International Automation.

[19] S. Of, H. Faults, I. N. Energyplus, Modeling and simulation of HVAC Results in EnergyPlus, IBPSA Building Simulation 14-16.

[20] M. Trcka, R. Khire, M. Gorbounov, Fault Model Library in TRNSYS for Failure Mode Effect Analysis on Whole Building Energy Perfor- 
mance MoGvaGon and ObjecGve, in: Re-energizing buildings of for the future, 2013.

[21] J. Hyvarinen, S. Arki, IEA Annex 25: Building optimisation and Fault diagnosis Source book, Espoo.

[22] D. Jump, L. Webster, M. Effinger, G. Risko, Guidelines for Verifying Savings from Commissioning Existing Buildings.

[23] M. Cherniack, Fault Detection and Diagnostics : Automated, Ongoing Commissioning Functionality for Large and Small HVAC Systems So Who s and What s at Fault? (2006).

URL http://www.bcxa.org/ncbc/2006/proceedings/17_ Cherniack_NCBC2006.pdf

[24] Z. Du, X. Jin, Y. Yang, Fault diagnosis for temperature, flow rate and pressure sensors in VAV systems using wavelet neural network, Applied Energy (9) 1624-1631. doi:10.1016/j.apenergy.2009.01.015.

[25] S. H. Kim, An evaluation of robust controls for passive building thermal mass and mechanical thermal energy storage under uncertainty, Applied Energy 602-623doi:10.1016/j.apenergy.2013.05.030.

[26] W. Fisk, O. Seppanen, Providing Better Indoor Environmental Quality Brings Economic Benefits, Clima 2007 Wellbeing Indoors (2007) 3-19.

[27] O. for economic co operation, develompent (OECD), Average annual wages over the world in USD (2013). URL http://stats . oecd.org//Index . aspx?QueryId=64115

[28] E. E. C. Bank), Inflation forecasts for the European union. (2016). 
URL https://www.ecb.europa.eu/stats/prices/indic/forecast/ html/table_hist_hicp.en.html

[29] H. Group, Wage prognosis in the UK. (2015). URL http://www . haygroup.com/uk/press/Details . aspx?ID=45743

[30] U. of Wisconsin, Trnsys, a transient simulation program, Thermal Energy System Specialists (TESS), Madison, Wis, 1976.

[31] MATLAB, version 7.10.0 (R2010a), The MathWorks Inc., Natick, Massachusetts, 2010.

[32] T. Van Den Brande, Master Thesis (Dutch): Evaluatie van modelgebaseerde regelaars door dynamische gebouwsimulaties met Energy Plus, Master thesis, KULeuven (2015).

[33] W. Parys, Cost optimization of cellular office buildings based on building energy simulation. Ph.D, no. April 2013.

[34] J. Lofberg, YALMIP : A Toolbox for Modeling and Optimization in MATLAB. , Proceedings of the CACSD Conference, Taipei, Taiwan.

[35] G. optimisation, Gurobi solver (2015).

URL www.gurobi.com

[36] J. Verhelst, J. V. der Veken, G. V. Ham, Tuning of Control Parameters by Combining Building Simulations and a Multi-Magnitude Wireless Sensor Network: A Case Study, ceae.colorado.edu 1273-1282. 


\section{Appendix: Case study description}

\subsection{Office building and HVAC emulator models}

Several distinct software tools are available to simulate HVAC, faults and model based control. However, software tools that can simulate the combination of detailed HVAC behaviour, fault effects and model predictive control in detail, are more scarce. Suitable HVAC emulation packages capable of the level of HVAC detail investigated in this paper, include (aside from Trnsys): E+, HVACsim, EES, BLAST, DOE2 and Modelica. Faulty signals can be modeled in E+, HVACsim, EES and Modelica. MPC control interfaces with emulation software can be implemented by coupling solvers (e.g. CPLex, Gurobi) through middleware software (e.g. Python, Matlab, BCVTB).

In this paper, the investigated cases and faults are modelled using Trnsys (30), while the HVAC-control is implemented in Matlab (31). Thus a TrnsysMatlab coupling was required. For this purpose, Trnsys type 155 was used. As an exercise, the possibilities of applying supervisory MPC-control to a E+-emulation have been investigated and implemented in the framework of a master thesis (32), using E+ as an emulator with CPlex as solver, coupled through middle-ware BCVTB and Matlab.

A building construction with high thermal inertia and with thick thermally activated building system (TABS) consisting of concrete construction elements (concrete core activation, CCA) are assumed here. The used material properties are shown in table 10.

A schematic representation of the HVAC system is shown in figure 11 . For baseload cooling and heating, concrete core activation (CCA) tubes are included (symmetrically) in the floor and ceiling sections of this office 


\begin{tabular}{c|cc}
\hline Property & Value & Units \\
\hline Office section width & 3.00 & $\mathrm{~m}$ \\
Office section depth & 4.00 & $\mathrm{~m}$ \\
Floor to floor height & 2.9 & $\mathrm{~m}$ \\
Floor to plenum height & 2.4 & $\mathrm{~m}$ \\
Glass to wall ratio & 50 & $\%$ \\
Orientation & $\mathrm{N}-\mathrm{S}$ and E-W & - \\
\hline
\end{tabular}

Table 9: Emulated building layout

\begin{tabular}{c|c|cc|cc}
\hline $\begin{array}{c}\text { Component } \\
\text { Units }\end{array}$ & adjoining: & $\begin{array}{c}\text { Thickness } \\
{[\mathrm{m}]}\end{array}$ & $\begin{array}{c}\text { Area } \\
{\left[\mathrm{m}^{2}\right]}\end{array}$ & $\begin{array}{c}\text { Thermal cond. U } \\
{\left[W / \mathrm{m}^{2} / \mathrm{K}\right]}\end{array}$ & $\begin{array}{c}\text { Accessible Capacity } \\
{[\mathrm{kJ} / \mathrm{K}]}\end{array}$ \\
\hline External wall & ambient & - & 8.64 & 0.412 & 3594 \\
Internal wall & Corridor & - & 7.2 & 0.790 & 69 \\
Internal wall & office & - & 11.6 & Adiabatic & Adiabatic \\
Floor & lower floor & - & 12 & Adiabatic & 6873 \\
Ceiling & upper floor & - & 12 & Adiabatic & 6873 \\
Window & office & - & 4.32 & 1.09 (g: 0.39$)$ & - \\
\hline
\end{tabular}

Table 10: Building layer build-up

\begin{tabular}{c|c}
\hline Building mass & Heavy \\
\hline Cooling production system & idealized chiller \\
Production Cooling capacity & $100 \mathrm{~W} / \mathrm{m}^{2}$ \\
Cooling emission system & $\mathrm{CCA}$ \\
\hline Heating production system & idealized boiler \\
Production Heating capacity & $105 \mathrm{~W} / \mathrm{m}^{2}$ \\
Heat emission system & $\mathrm{CCA}$ \\
Ventilation & $\mathrm{CAV}$ \\
Climate & TMY2 Uccle, Belgium \\
\hline
\end{tabular}

Table 11: HVAC sizing and emulator limitations 
building. These are modelled using the EMPA model (7) embedded in an active building layer, with a fixed flow rate of $14 \mathrm{~kg} / \mathrm{h} / \mathrm{m}^{2}$ office area (or 12 $\mathrm{kg} / \mathrm{h} / \mathrm{m}^{2}$ gross floor area) during operation. The specified tube diameter is $0.02 \mathrm{~m}$, with a wall thickness of $0.002 \mathrm{~m}$ and a pipe spacing (centre to centre) of $0.15 \mathrm{~m}$. The assumed pipe wall conductivity is $1.26 \mathrm{~kJ} / \mathrm{h} / \mathrm{m} / \mathrm{k}$. The pipes are embedded in the centre of a $20 \mathrm{~cm}$ concrete slab, with a $0.075 \mathrm{~m}$ screed layer, an air layer (raised computer floor) and $0.04 \mathrm{~m}$ thick floor-tiles on top of it. The ceiling is covered with a $0.01 \mathrm{~cm}$ plaster layer.

The water distribution system consists out of two supply collectors (a hot water and cold water collector) and several three way valves, allowing each zone to be independently cooled or heated and have a separate, unique supply setpoint and flow rate. The water supply setpoint for each zones is set every hour, by the supervisory controller. Furthermore, each supervisory control strategy has the ability to override the flow rate towards the HVAC emitters for each zone separately.

The installed hot and cold water production capacity for this water based heating and cooling emission system are provided by a central boiler and a central chiller. These are selected with a maximal production capacity of respectively $100 \mathrm{~W} / \mathrm{m}^{2}$ for heating and $105 \mathrm{~W} / \mathrm{m}^{2}$ for cooling. These figures are based on a steady heat load calculation of the building at $-8{ }^{\circ} \mathrm{C}$ for heating and $30^{\circ}$ for cooling, with an additional start-up margin of 200 $\%$.

While the efficiency of a real HVAC production system is typically not constant, but dependent on boundary conditions and installation, in this study this conservative assumption is made to eliminate differences in control performance between different operational strategies which may exploit production efficiency information. Therefore, it allows us to pinpoint solely 
the fault impacts due to changes in emission-, control- and distribution efficiencies. A fixed production conversion efficiency (independent of supply temperature or part load ratio) is modelled with a seasonal efficiency ratio for heating ( $\mathrm{sCOP}$ ) of 0.9 for heating, and seasonal efficiency ratio for cooling (sEER) equal to 2.5 .

As ventilation system, a constant air volume (CAV) ventilation system is modelled, with scheduled, fixed supply air flow rates of $43.2 \mathrm{~kg} / \mathrm{h} / \mathrm{m}^{2}$. In the modelled AHU, a thermal wheel with a sensible efficiency of $80 \%$, a heating coil and a cooling coil are present. No humidification or active dehumidification is performed.

Humidity levels of zone air and supply air are not actively controlled or verified in this emulator. Modelling realistic humidity dynamics in detail in the emulator model would require a full hydrothermal model based on a buffer storage model, requiring additional (unavailable) information on the hygrodynamics of building materials and furniture in the room to determine realistic parameter values for e.g. gradients of sorption and moisture exchange coefficients. This is out of scope of this study as the focus is on thermal comfort.

Some humidity dynamics are however taken into account in the current model (in a simplified manner). A simple capacitance humidity model is used, with a humidity capacitance ratio of 1 for both zones. Also, some (indirect) dehumidification of the ventilation air is performed. A cooling coil is modelled in the AHU, which cools down ambient air to a target supply temperature setpoint following a heat curve. The cooling coil outputs fresh air with a maximal wet bulb temperature of $19-24{ }^{\circ} \mathrm{C}$.

Outside of scheduled occupancy, zone air is recirculated and during occupancy, $100 \%$ fresh air is supplied, pretreated by the thermal wheel, cooling 
coil and heating coil, to the desired air supply setpoint $T_{s a}$.

Infiltration is modelled to be $0.05 \mathrm{ACH}$ when the ventilation system is active, $0.2 \mathrm{ACH}$ per hour when ventilation system is off.

The air supply setpoint can be set independently for both zones. The RBC provides preheated and precooled fresh air at a supply air setpoint for both zones of $24{ }^{\circ} \mathrm{C}$ during winter, and $19{ }^{\circ} \mathrm{C}$ during summer conditions, with a changeover condition determined based on the mean outside temperature $\left(15{ }^{\circ} \mathrm{C}\right)$. The other control algorithms can vary the supply temperature between 15 and $30{ }^{\circ} \mathrm{C}$.

For occupancy and internal gains, a standardized ASHRAE occupancy time schedule with 1 occupant per $10 \mathrm{~m}^{2}$ of office space is modeled, between $8 \mathrm{AM}$ and $12 \mathrm{AM}$ and $1 \mathrm{PM}$ to $6 \mathrm{PM}$ during week days, to resemble a characteristic landscape office occupancy. Corresponding internal sensible heat gains of $75 \mathrm{~W} /$ person and $55 \mathrm{~W} /$ per son latent heat gains are assumed, for seated occupants doing light work. The sensible heat gains are split into convective gains (42\%) and radiative heat gains (58\%).

As weather disturbance, the TMY2 weather file for Uccle (Belgium) has been applied to the building. A solar shading system with hysteresis is programmed for the south side, triggered based on two threshold levels of incident solar irradiation on that facade.

For comfort evaluations, a target comfort band with a width of $4{ }^{\circ} \mathrm{C}$ is used, typically between 20 and $24{ }^{\circ} \mathrm{C}$, but this band is adjusted upwards or downwards slightly at extreme mean outdoor temperatures, based on the adaptive comfort theory. The HVAC emission systems have a target operative air setpoint equal to these boundaries, with an offset of $0.5{ }^{\circ} \mathrm{C}$ towards the middle to allow hunting without causing thermal discomfort.

This two-zone-office-CCA emulator model was designed from bottom up, 
using a white box methodology (based on building material properties and good practices). Therefore, no exact one-to-one mapping can be made to a specific existing building.

Researchers Maarten Sourbron and Wout Parys have successfully verified the white box parameters of this concrete core conditioned office building emulator; based on measured indoor temperatures and HVAC temperatures in an office building in Belgium, Hasselt, both in open loop operation with response to step inputs and in closed loop operation with a rule based controller and also with an MPC implementation. They verified cool-down and heat-up temperature dynamics of measurements and simulation output under the influence of different disturbances. The model and procedure are described in detail in their Phd theses (? ) and (33) (appendix A).

\subsection{Supervisory HVAC-Controller implementations}

Four model based controller types are developed, a RBC, OL-MPC, CLMPC and R-MPC. In following section, the used control algorithms and control implementation are described for each controller.

\subsection{1. $R B C$}

As a reference supervisory controller, a rule based heating and cooling curve (outdoor temperature reset) controller (RBC) is used. This controller takes the historic (multi-day weighted) outdoor air temperature as an input and calculates appropriate (concrete core actiation and ventilation) supply temperatures based on the heat curve equations 7. with $T_{s, w / a}$ the supply temperature of water or air respectively, $T_{a a}$ the historically weighted ambient air temperature $T_{s, w / a, \max }$ and $T_{s, w / a, \max }$ the supply temperature limits defined by the facility manager, based on equipment safety and efficiency, 


$$
\begin{aligned}
T_{s, w / a}=\min ( & T_{s, w / a, \max }, \max \left(T_{s, w / a, \min },\right. \\
& \left.\left.+B *\left(T_{\text {ref }}-T_{a a}\right)^{C}+T_{\text {Boost }} * I_{\text {boost }}-T \text { setback } * I_{\text {setback }}\right)\right)
\end{aligned}
$$

To find a near-optimally tuned RBC parameter values for parameters

A, B, $T_{r e f}$ for each circuit (w: water, a: air) for the fault-free case, a latin hypercube sampling was performed with 1000 year-simulations with varying parameter values. One heat curve was used for both zones. The parameters which lead to the lowest total energy and discomfort cost (with a weighting factor of 0.5 ) in the fault-free condition using the (simulated) dynamic response of the emulated building to ambient temperature over a reference year, are retained. The chosen $\mathrm{RBC}$ parameters are given in table 12 . The $C$-parameter was fixed at a value of 1 . The boost term (increased water supply temperature on Monday morning) and a weekend setback (widened set-point range) were found not to be required (non-optimal) for this case.

\subsection{2. $M P C s$}

The open loop MPC (OL-MPC) and closed loop MPC (CL-MPC) require an analytic dynamic system model of the emulator to minimise a cost function. Either a white box model, black box model or grey box model can be used for this purpose. In this case, a black box state space model is 


\begin{tabular}{l|l}
\hline Parameter & value \\
\hline$A_{s, w, c}$ & 27.9 \\
$A_{s, w, h}$ & 34.7 \\
$A_{s, a, c}$ & 15 \\
$A_{s, a, h}$ & 13 \\
$B_{s, w, h}$ & -0.22 \\
$B_{s, w, c}$ & -0.16 \\
$B_{s, a, h}$ & 1 \\
$B_{s, a, c}$ & 1 \\
$C$ & 1 \\
$T_{r e f, s u m m e r}$ & 15 \\
$T_{r e f, w i n t e r}$ & 13 \\
Boostw,h & 0 \\
Setbackw,h & 0 \\
\hline$T_{s, w, \text { min }}$ & 15 \\
$T_{s, w, \text { max }}$ & 45 \\
$T_{s, a, \text { min }}$ & 18 (15 for MPC) \\
$T_{s, a, \text { max }}$ & 20 (35 for MPC) \\
\hline
\end{tabular}

Table 12: Near-optimal heat curve parameters for RBC control and supply temperature limits used for RBC and MPC control simulation. . 
constructed. The identification process is executed in two stages:

First, a pseudo-random-multisine-excitation is applied to the main HVACcontroller inputs and disturbances: water supply temperature $T_{s, w}$, air supply temperature $T_{s, a}$, ambient air temperature $T_{a a}$, internal gains $Q_{i n t}$, irradiation $Q_{i r r}$ on one zone (S zone) of the Trnsys emulator (for each of the three building/HVAC cases). The response of following outputs is recorded: indoor air temperature $T_{z, a}$, return water temperature $T_{r w}$ and concrete core temperature $T_{c c}$. This identification data set is then split in an identification and cross-validation set, with the ratio of samples 10:1.Based on these data sets, a 4-state state space model is identified using the Matlab ident-toolbox . As identification settings, the N4SID-identification method is selected, with a focus on simulation and with a delay of 1 time step (12 minutes) for the inputs water supply temperature $T_{s, w}$ and air supply temperature $T_{s, a}$.

The second zone ( $\mathrm{N}$ zone) is assumed to have the same response to the input variables, as the building construction and HVAC system is identical. Therefore, the same model is used to control the north and the south zone in the model based controller, both during summer and winter.

As a cost function, a linear cost function (equation 8) is used, consisting of the weighted sum of discomfort cost (underheating and undercooling) and energy cost (water climatization and ventilation), using the parameter values given table 12. Hard constraints are applied to limit the supply temperatures and production heat fluxes. Slack variables are introduced and paired with the operative zone temperature to determine the level of thermal discomfort.

The problem structure, constraints and cost function are expressed using the Yalmip toolbox (34). As a solver, Gurobi (35) was used to solve all 
linear, time invariant MPC problems. This cost-function is minimised over the prediction horizon by the MPC controllers for each zone separately. From the optimal result, the requested HVAC temperature setpoints for the production side and zone temperature control valves are obtained.

$$
\begin{aligned}
& \min _{z, t}=(W) *\left(\overline{C_{w, t}+C_{a, t}}\right)+(1-W) * \overline{C_{d, t}} \\
& \text { s.t. } T_{w / a, \min }<T s, w / a, t<T_{w / a, \max } \\
& P_{w / a, \min }<P s, w / a, t<P_{w / a, \max } \\
& C_{d, t}=\max \left(\left\|T_{\text {comf }, \min }-T a, z, t\right\|, \ldots\right. \\
& \left.\left\|T_{\text {comf }, \max }-T a, z, t\right\|, 0\right) * t * C_{d i s c} \\
& C_{w / a, t}=\left(T_{s, w / a}-T r, w / a\right) * c_{p, w / a} * t * C_{E, w / a}
\end{aligned}
$$

For the OL-MPC, for each zone an (open loop) optimization problem with an horizon of one year is defined and pre-calculated. The control actions are then applied to the building, with only low feedback control level-adjustments.

For the CL-MPC supervisory controller, a similar approach is used, but with a recurring estimation, and a prediction horizon of only 48 hours for each optimization. State estimation is performed using historic measurements and inputs over a backward horizon of 24 hours. At every control time-step, only calculated control inputs of the control horizon (6 hours) are applied. The choice of a prediction horizon of 48 hours is supported by the dominant frequencies in the building, identified using a step response. The control horizon of 6 hours is chosen to speed up the simulations, compared to a control horizon of 1 hour. The same constraints, quadratic cost function and weights are used for this closed loop controller and for the open loop 
controller. Due to different horizon length, state estimation and model mismatch, however, this results in a slightly different yearly trade-off between energy cost and discomfort cost compared to the open loop performance.

For the R-MPC supervisory controller, the same model structure and horizons are used as for the CL-MPC (the sum of energy use and discomfort is minimized), but bounded additive uncertainty on the input and output are assumed on all faulty signals (sensors and actuators), equal to the light additive fault severity level. A min-max MPC scheme is used, where both the discomfort and energy use in the worst case for every timestep (max), are minimized ( $\min$ ) over the whole prediction horizon. As the building model has positive state-space matrices and the control inputs are all positive (temperatures and heat fluxes), this min-max MPC can be implemented using an envelope method with the extreme values of the uncertain parameters. This way, a stochastic R-MPC problem is simplified to a non-stochastic, linear MPC problem, which still takes into account the (worse case) additive uncertainty.

\subsubsection{Controller implementation}

For the RBC reference controller and the OL-MPC controller, calculated supply temperatures are applied to the emulator in a feed forward manner without any direct feedback from the HVAC system or zone temperature.

On the other hand, both the CL-MPC and the R-MPC follow the receding horizon control principle, with historic output and input feedback. A receding horizon of $6 \mathrm{~h}$ is used, with a control timestep of $1 \mathrm{~h}$. Every $6 \mathrm{~h}$, the controller output is recalculated based on latest outputs from the building and the first part (6 hs) of the calculated control output is applied to the building. No prediction errors are introduced to the MPC controllers: Over 
the MPC model prediction horizon, the future solar irradiation, ambient temperature and occupancy patterns are assumed to be predicted perfectly, even if a sensor fault is present. No Kalman filtering is applied, but state estimation is performed based on the measured historic outputs and historic control inputs.

\subsubsection{Competing control objectives}

Indoor air acclimatization settings mainly depend on the chosen tradeoff between discomfort cost and HVAC running costs. The HVAC running cost contains mainly energy cost, but might include maintenance cost and commissioning costs. Also, (one time) case-study- or controller-dependent investment costs may be included in the comparisons.

For RBC, the weights of this trade-off cannot be defined explicitly within the control model, but the resulting trade-off can be shifted through nontrivial adjustment of the RBC parameters. In this emulation case, the heat curve reference temperature $A$ and heat curve slope $B$ are varied to evaluate alternative reachable trade-off points for the reference controller in fault-free conditions. The most optimal (lowest energy and discomfort cost) combination of multiple random heat curves runs are (assumed) to be on the Pareto-front reachable by this controller. Especially the heat curve slope $B$ of the heating curve has a highly (non)-linear influence on yearly energy use and discomfort (36).

For MPC (OL-MPC, CL-MPC and R-MPC), a trade-off parameter can be put explicitly in the cost function over the control horizon, but when evaluated on yearly bases, the resulting KPI trade-off will not exactly equal this preset value, as it also depends on the model mismatch, prediction mismatch, equipment sizing and the persistent faults present in the building. 
If the weight $\alpha$ placed on energy cost is 1 , only the energy cost will be minimized by the control algorithm, resulting in a temperature profile comparable to a free running system (no energy use). If the weight placed on energy use $\alpha$ is 0 , this corresponds to minimizing the cost related to discomfort, meaning the controller aims for a perfect comfort control, at any (potentially very high) HVAC-related energy cost.

In these simulations, the OL-MPC and R-MPC trade-off weight $\alpha$ are chosen (a-posteriori) to be 0.5 , while the CL-MPC weight is empirically fixed at 0.908

[1] S. Katipamula, M. Brambley, Review Article: Methods for Fault Detection, Diagnostics, and Prognostics for Building SystemsA Review, Part II, HVAC\&R Research 11 (2) (2005) 169-187. doi:10.1080/10789669. 2005.10391133.

[2] N. Djuric, Real-time supervision of building HVAC system performance, Ph.D. thesis, Norwegian Univrsity of Science and technology (2007).

[3] G. Huang, F. Jordán, Model-based robust temperature control for VAV air-conditioning system, HVAC and R Research (3) 432-445. doi: 10.1080/10789669.2012.649879.

[4] S. Katipamula, M. Brambley, Review Article: Methods for Fault De-

\footnotetext{
${ }^{8}$ When the CL-MPC trade-off weight $\alpha$ is set to 0.5 , the CL-MPC controller, when applied to the emulator with limited prediction horizon $(48 \mathrm{~h})$, it was able to aa slightly better comfort situation, but used a lot more energy than the other controllers to avoid discomfort. By adjusting the weighting factor $\alpha$ to 0.95 , this trade-off is shifted towards lower energy use and minimal total cost in fault-free conditions.
} 
tection, Diagnostics, and Prognostics for Building SystemsA Review,

7. Part I, HVAC\&R Research (1) 3-25. doi:10.1080/10789669.2005. 10391133 .

[5] S. Wu, K. Neale, M. Williamson, M. Hornby, Research opportunities in maintenance of office building services systems, Journal of Quality in Maintenance Engineering 16 (1) (2010) 23-33.

[6] W. Gntensperger, M. Gwerder, A. Haas, B. Lehmann, F. Renggli, J. Tdtli, Control of concrete core conditioning systems , Proceedings of the 8th REHVA World Congress for Building Technologies CLIMA 2005.

[7] M. Sourbron, Dynamic thermal behaviour of buildings with concrete core activation, Ph.D. thesis.

[8] K. Roth, D. Westphalen, M. Feng, The Energy Impact of Faults in U.S. Commercial Buildings, International Refrigeration and Air Conditioning Conference. Paper 665.

[9] S. Li, A Model-Based Fault Detection and Diagnostic Methodology for Secondary HVAC Systems, Drexel University.

[10] K. J.Y., E. Pierce, Sensor errors and their effecto $n$ building energy consumption, ASHRAE 25.

[11] S. Prívara, Building modeling and identification for predictive control, Ph.D. thesis, Czech Technical university in prague.

[12] M. Maasoumy, Controlling Energy-Efficient Buildings in the Context of the Smart Grid : A Cyber-Physical System Approach Smart Build- 
1074

ings in the Smart Grid, Ph.D. thesis, University of California, Berkeley (2013).

[13] M. Trčka, J. L. M. Hensen, Overview of HVAC system simulation, Automation in Construction (2) 93-99. doi:10.1016/j.autcon.2009. 11.019 .

[14] D. Gyalistras, Final report Use of Weather and Occupancy Forecasts for Optimal Building Climate Control (OptiControl) 1. Management Summary (July).

[15] S. C. Bengea, A. D. Kelman, F. Borrelli, R. Taylor, Model Predictive Control for Mid-Size Commercial Building HVAC : Implementation , Results and Energy Savings, HVAC\&R Research 20 (2014) 979-986.

[16] M. Castilla, J. Álvarez, M. Berenguel, F. Rodríguez, J. Guzmán, M. Pérez, A comparison of thermal comfort predictive control strate․ gies, Energy and Buildings (10) 2737-2746. doi:10.1016/j.enbuild. 2011.06.030.

[17] T. S. Haves P., J. Wright, Condition monitoring in HVAC subsystems using first principles models, ASHRAE Transactions 102.

[18] J. E. Braun, R. W. H. Laboratories, Automated fault detection and diagnostics for vapor compression cooling equipment, International $\mathrm{Au}-$ tomation.

[19] S. Of, H. Faults, I. N. Energyplus, Modeling and simulation of HVAC Results in EnergyPlus, IBPSA Building Simulation 14-16.

[20] M. Trcka, R. Khire, M. Gorbounov, Fault Model Library in TRNSYS for Failure Mode Effect Analysis on Whole Building Energy Perfor- 
mance MoGvaGon and ObjecGve, in: Re-energizing buildings of for the future, 2013.

[21] J. Hyvarinen, S. Arki, IEA Annex 25: Building optimisation and Fault diagnosis Source book, Espoo.

[22] D. Jump, L. Webster, M. Effinger, G. Risko, Guidelines for Verifying Savings from Commissioning Existing Buildings.

[23] M. Cherniack, Fault Detection and Diagnostics : Automated, Ongoing Commissioning Functionality for Large and Small HVAC Systems So Who s and What s at Fault? (2006). URL http://www.bcxa.org/ncbc/2006/proceedings/17_ Cherniack_NCBC2006.pdf

[24] Z. Du, X. Jin, Y. Yang, Fault diagnosis for temperature, flow rate and pressure sensors in VAV systems using wavelet neural network, Applied Energy (9) 1624-1631. doi:10.1016/j.apenergy.2009.01.015.

[25] S. H. Kim, An evaluation of robust controls for passive building thermal mass and mechanical thermal energy storage under uncertainty, Applied Energy 602-623doi:10.1016/j.apenergy.2013.05.030.

[26] W. Fisk, O. Seppanen, Providing Better Indoor Environmental Quality Brings Economic Benefits, Clima 2007 Wellbeing Indoors (2007) 3-19.

[27] O. for economic co operation, develompent (OECD), Average annual wages over the world in USD (2013). URL http://stats . oecd . org//Index . aspx?QueryId=64115

[28] E. E. C. Bank), Inflation forecasts for the European union. (2016). 
URL https://www.ecb.europa.eu/stats/prices/indic/forecast/ html/table_hist_hicp.en.html

[29] H. Group, Wage prognosis in the UK. (2015). URL http://www . haygroup.com/uk/press/Details . aspx?ID=45743

[30] U. of Wisconsin, Trnsys, a transient simulation program, Thermal Energy System Specialists (TESS), Madison, Wis, 1976.

[31] MATLAB, version 7.10.0 (R2010a), The MathWorks Inc., Natick, Massachusetts, 2010.

[32] T. Van Den Brande, Master Thesis (Dutch): Evaluatie van modelgebaseerde regelaars door dynamische gebouwsimulaties met Energy Plus, Master thesis, KULeuven (2015).

[33] W. Parys, Cost optimization of cellular office buildings based on building energy simulation. Ph.D, no. April 2013.

[34] J. Lofberg, YALMIP : A Toolbox for Modeling and Optimization in MATLAB. , Proceedings of the CACSD Conference, Taipei, Taiwan.

[35] G. optimisation, Gurobi solver (2015).

URL www.gurobi.com

[36] J. Verhelst, J. V. der Veken, G. V. Ham, Tuning of Control Parameters by Combining Building Simulations and a Multi-Magnitude Wireless Sensor Network: A Case Study, ceae.colorado.edu 1273-1282. 

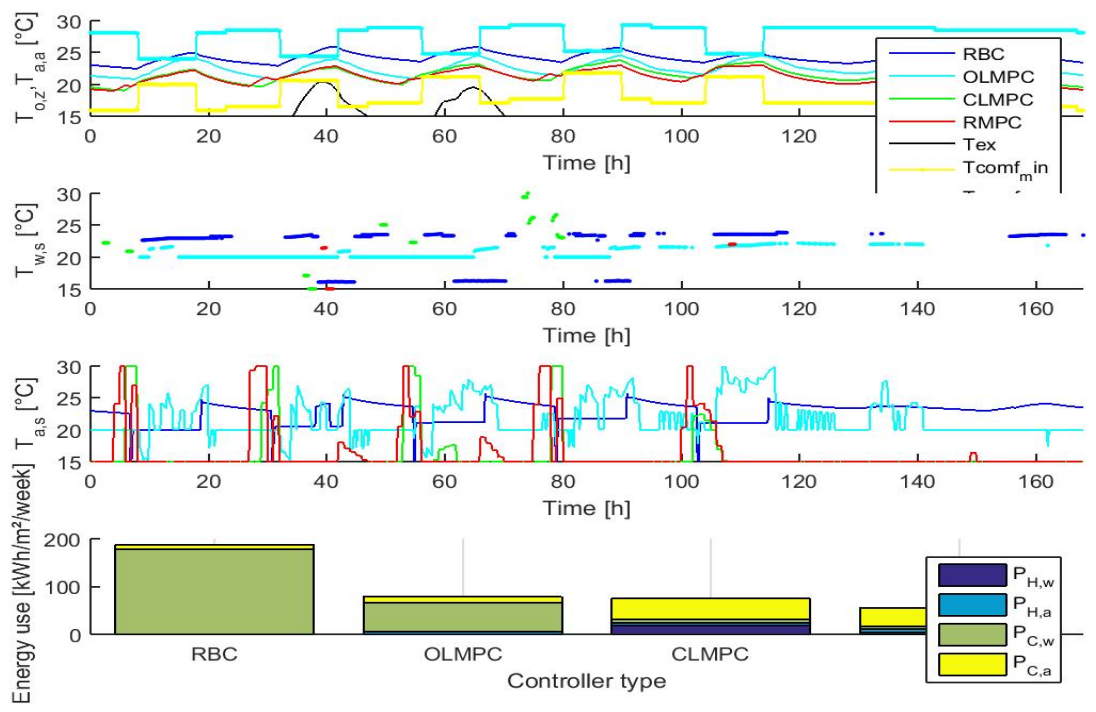

Figure 1: Simulated HVAC performance over one spring week, for four different controller types, in a fault free (FF) situation. In the top sub-figure, the bottom (black) line depicts the ambient temperature, the straight lines depict the comfort band and the four other lines show the operational zone temperatures achieved by different control strategies. Pane 2 and 3 contain the water and air supply temperature set by the different control strategies respectively. Pane 4 contains the resulting weekly energy usage. 


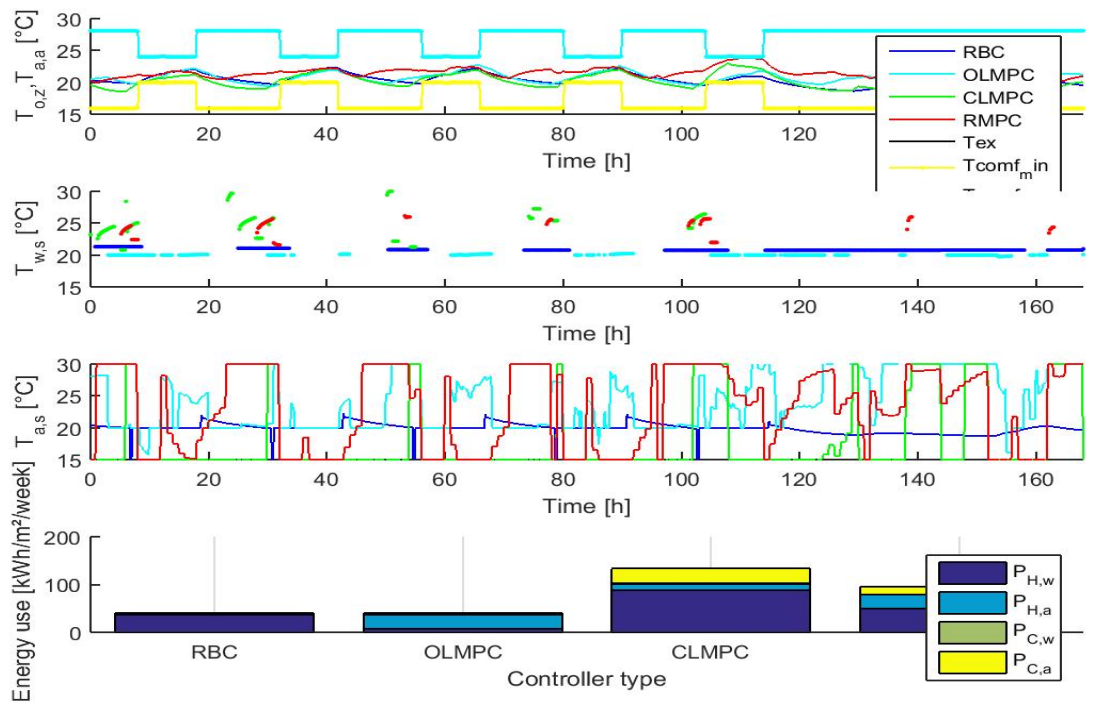

Figure 2: Simulated HVAC performance over one winter week, for four different controller types, in a fault free (FF) situation. In the top sub-figure, the bottom (black) line depicts the ambient temperature, the straight lines depict the comfort band and the four other lines show the operational zone temperatures achieved by different control strategies. Pane 2 and 3 contain the water and air supply temperature set by the different control strategies respectively. Pane 4 contains the resulting weekly energy usage. 


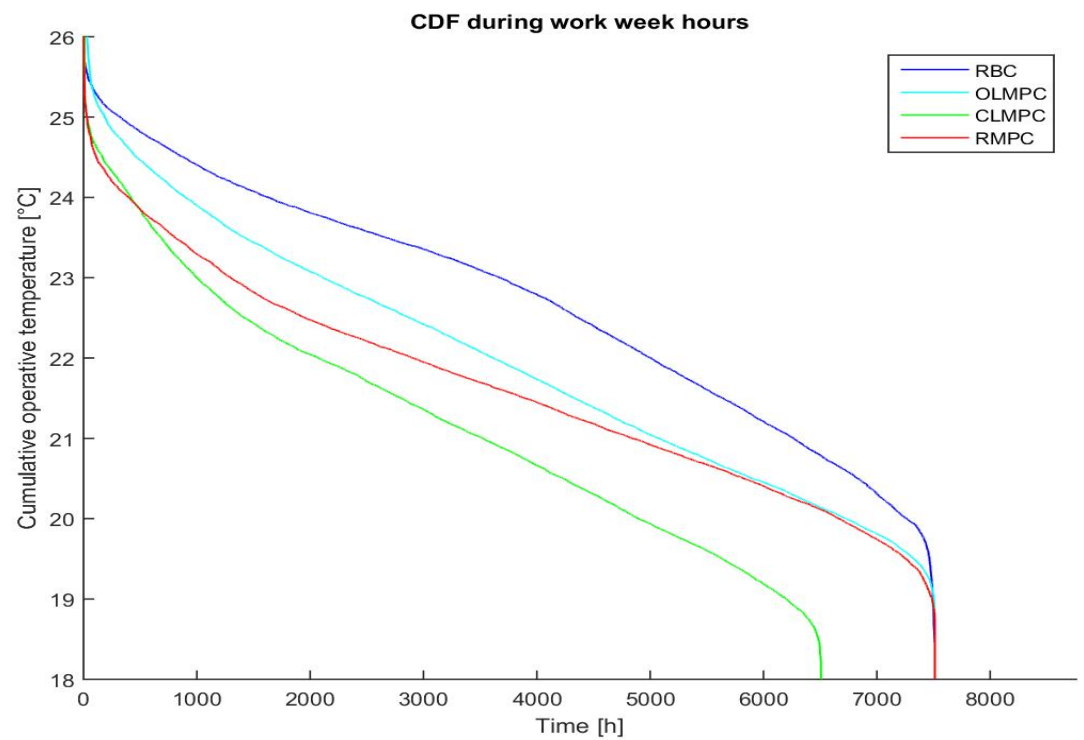

Figure 3: Simulated operative zone temperature duration curve for different CT with chosen weights, for the South zone of the emulated H CCA building case, in a FF situation, during office hours. . 


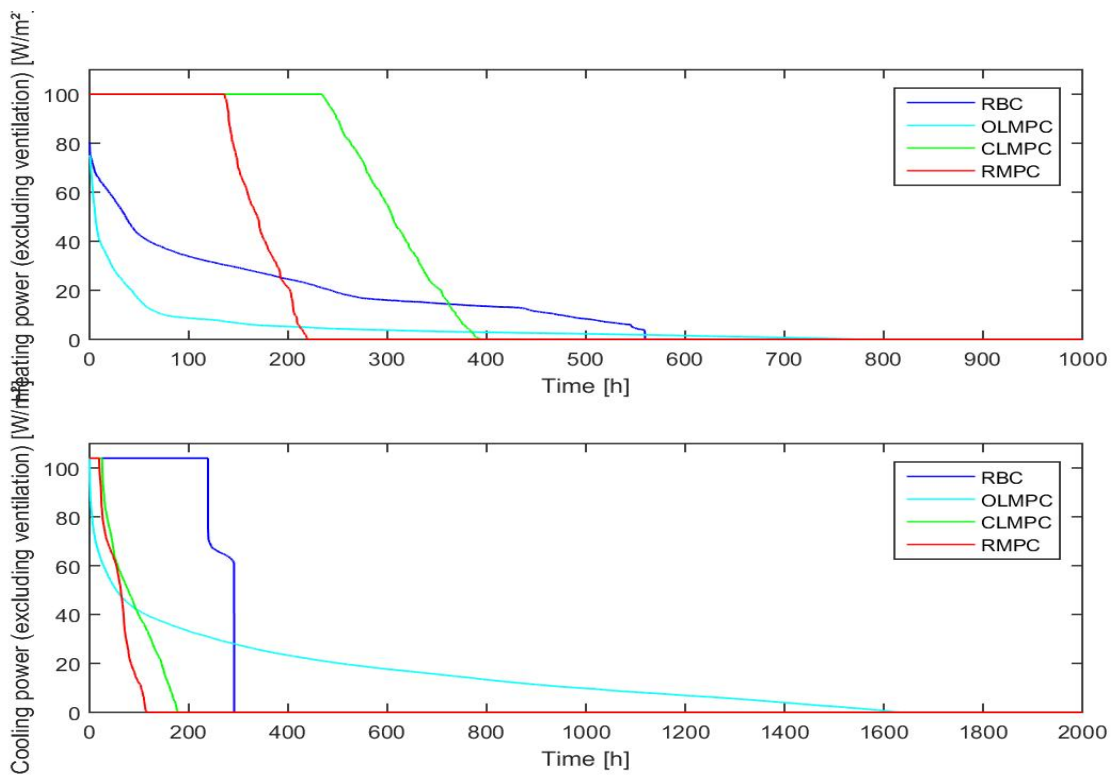

Figure 4: Production load duration curve of the heat and cold production for different controller types with fixed weights, in fault-free conditions, both for heating (top graph) and cooling (bottom graph). 

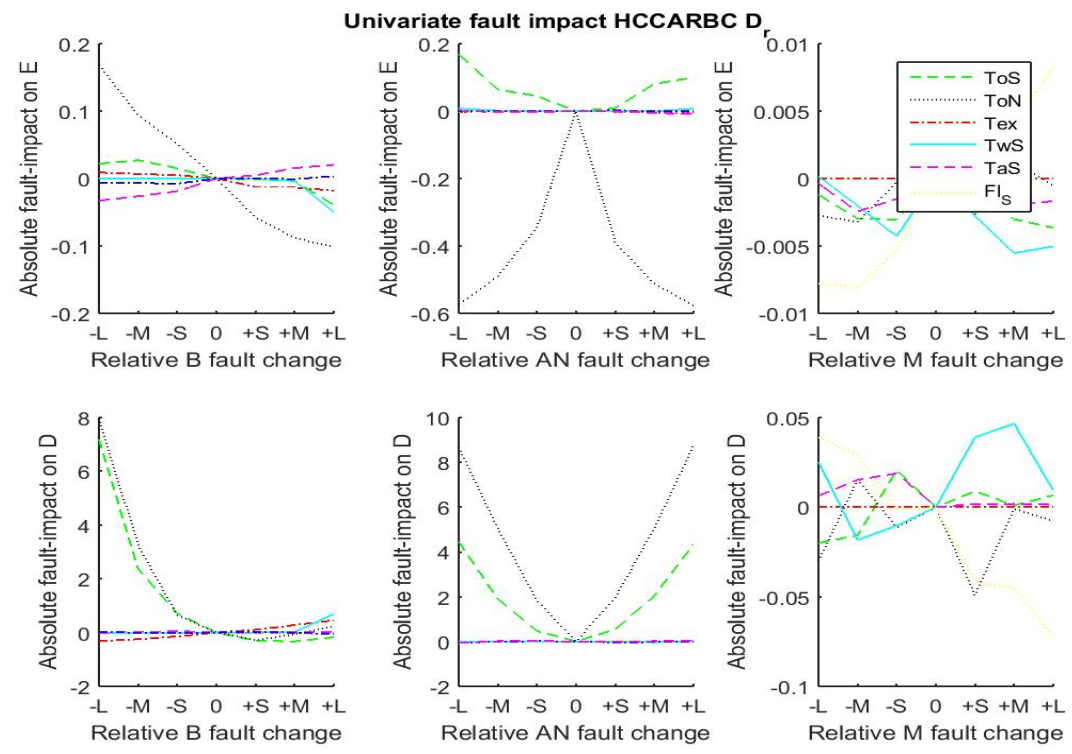

Figure 5: The absolute impact of single fault occurrence on energy cost [€/y] (top) and discomfort (bottom) cost $[€ / y]$ as a function of type of fault (FT), grouped by sensor component controlled by a Rule Based Controller (RBC). 

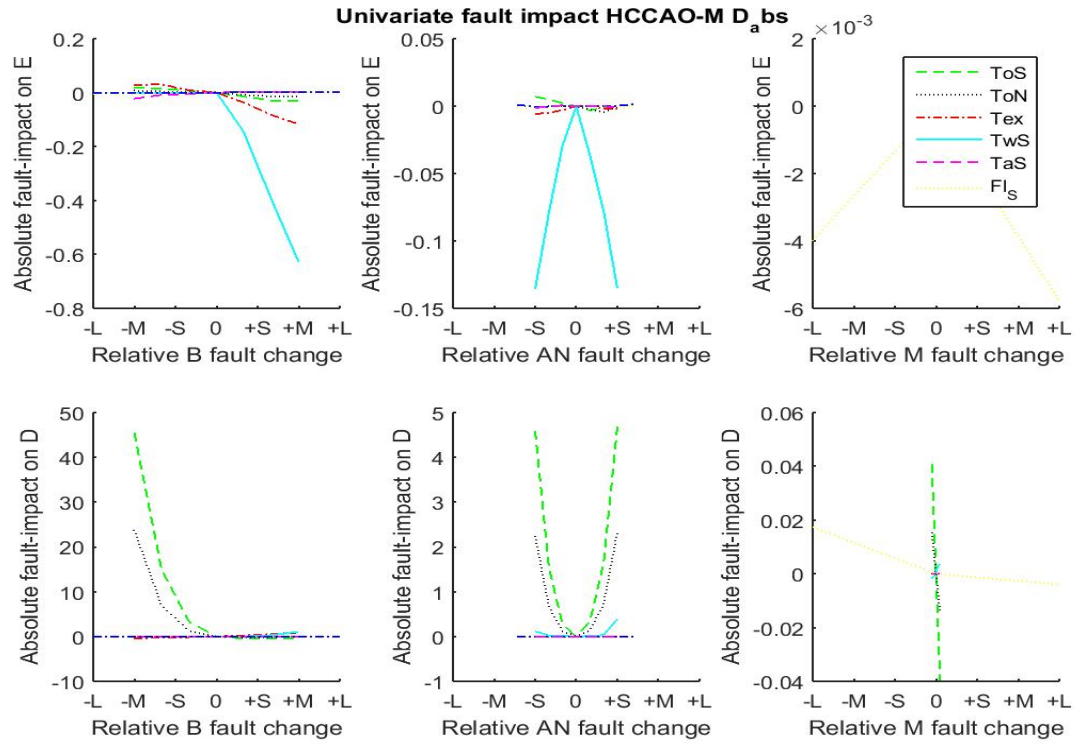

Figure 6: The absolute impact of single fault occurrence on energy cost [€/y] (top) and discomfort (bottom) cost $[€ / y]$ as a function of type of fault (FT), grouped by sensor component controlled by an open loop model predictive controller (OL-MPC). 


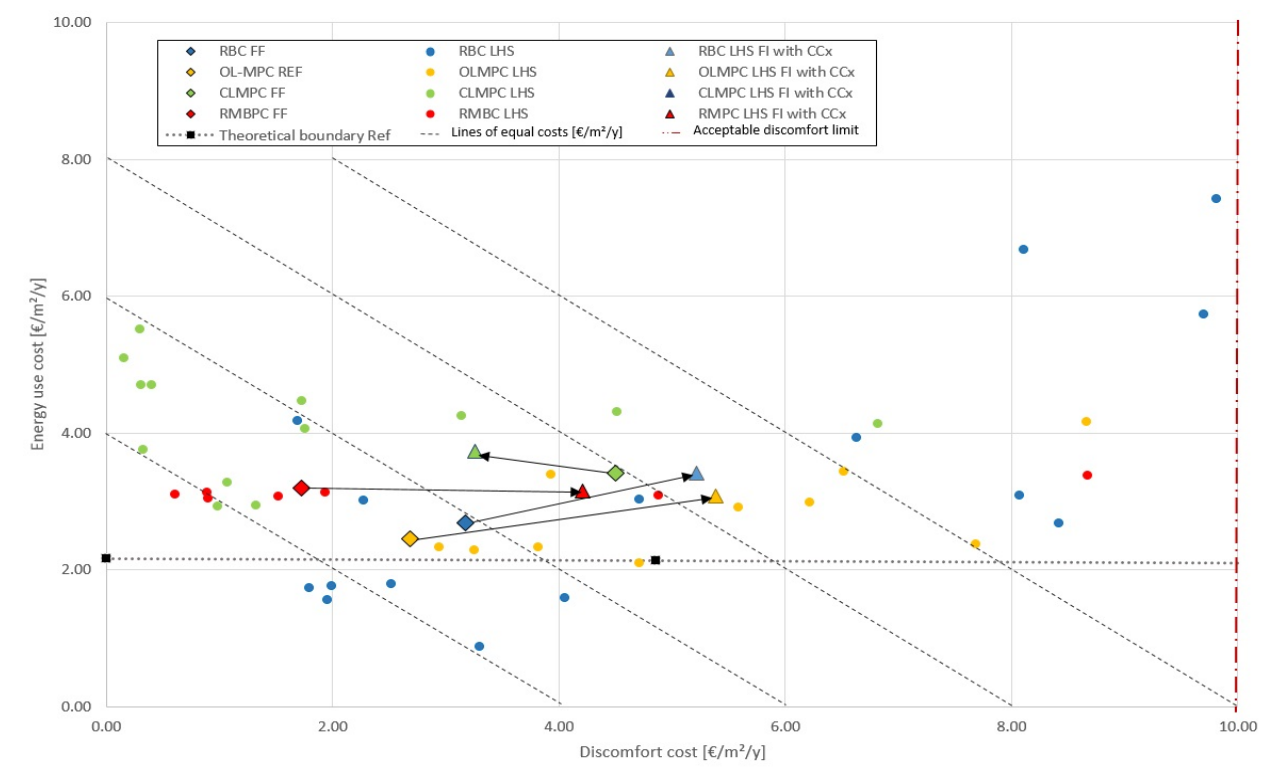

Figure 7: The impact of combined (LHS) fault occurrence on energy use and discomfort, for each of the four controller types. The reference (fault-free) performances (one point per controller) are annotated with diamonds. The weighted mean values, taking into account commissioning when low comfort situations occur, are depicted by triangles. . 


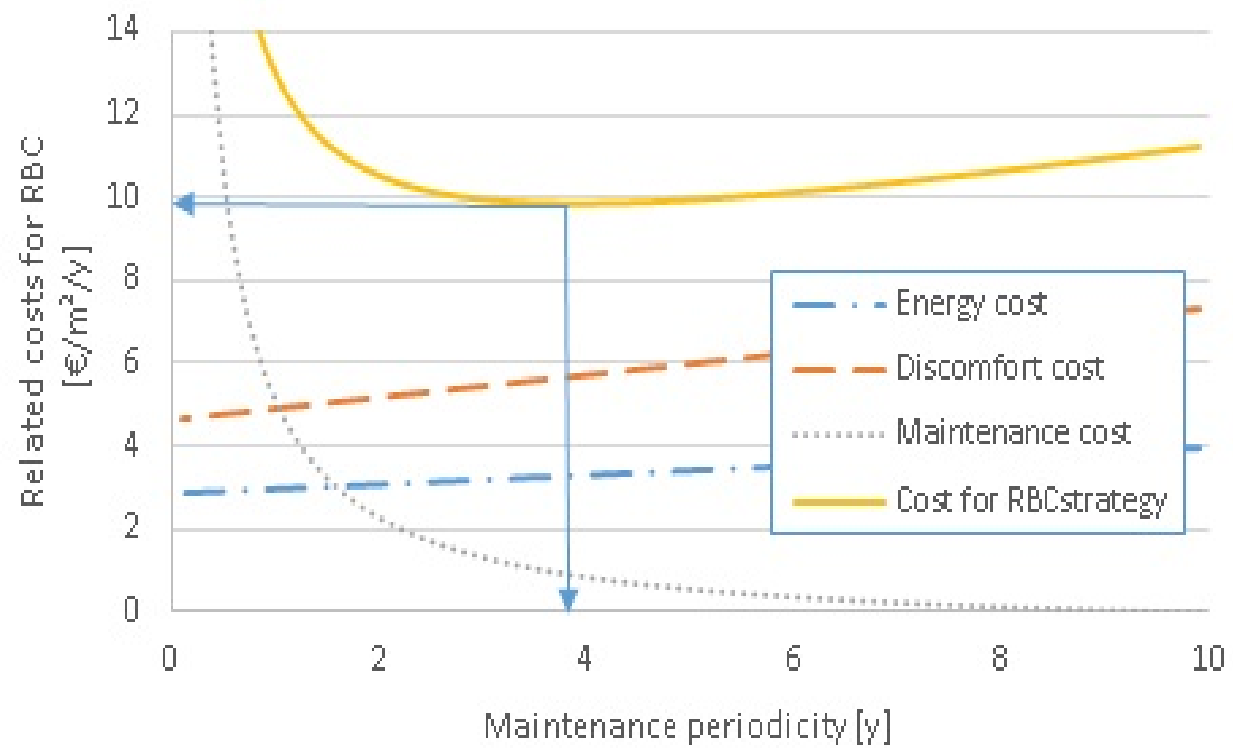

Figure 8: Total cost associated costs in function of maintenance periodicity $\left(\beta^{*} 10\right)$, together with the constituents energy cost, discomfort cost and maintenance cost. . 


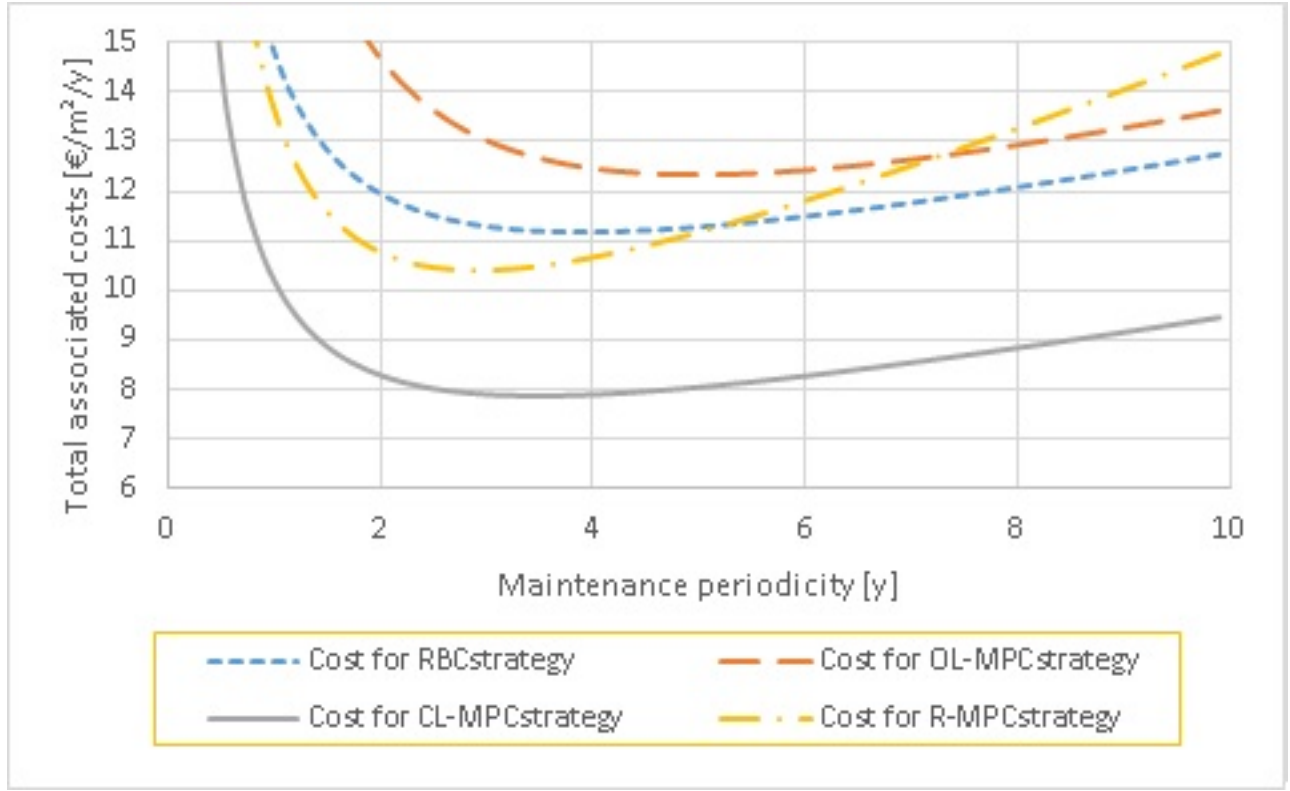

Figure 9: Total cost associated costs for the four evaluated controller strategies in function of maintenance periodicity $\left(\beta^{*} 10\right)$. The differences occur due to differences in reference cost, fault impact and high discomfort occurrence. 


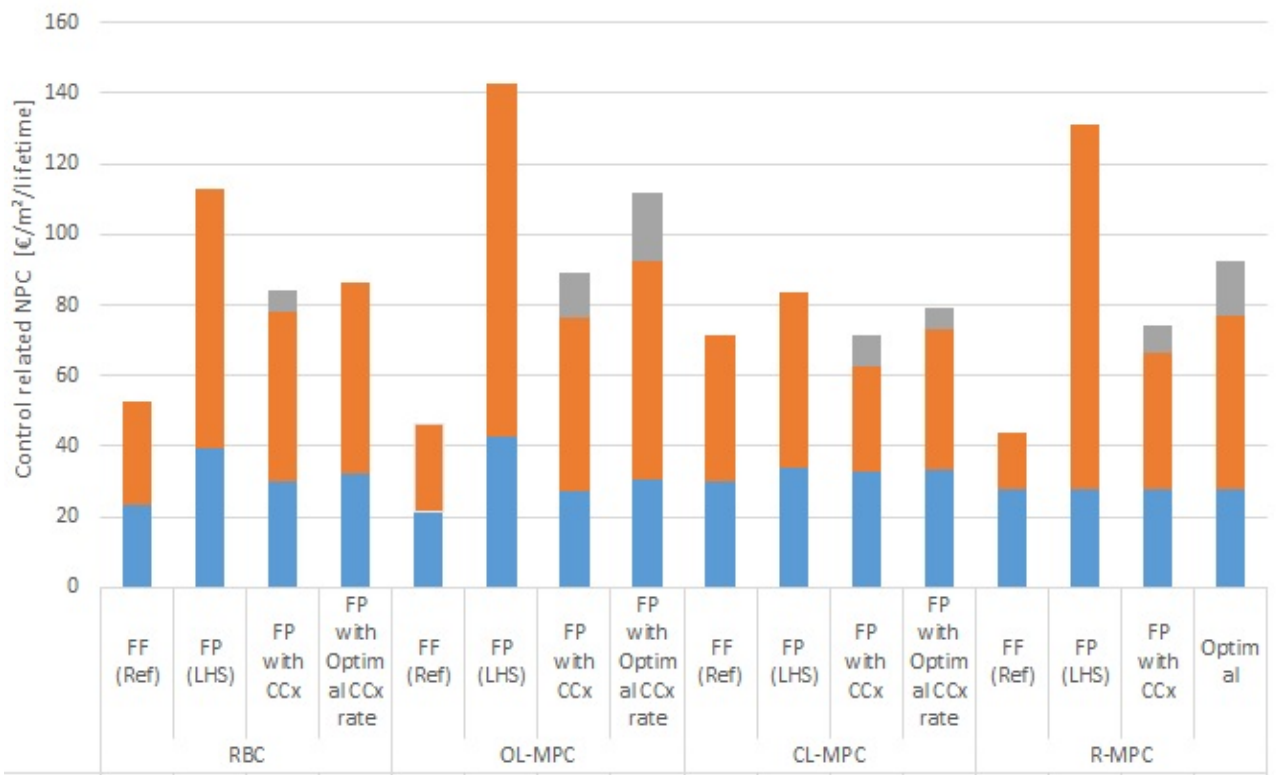

Figure 10: Overview of the three PC-constituents energy cost (bottom, blue bar), discomfort cost (middle, orange bar) and maintenance cost (top, grey bar) for each controller, in fault free (FF) conditions, under the presence of faults (FP) without commissioning, with regular commissioning $(\mathrm{CCx})$ and with optimal commissioning rate. . 


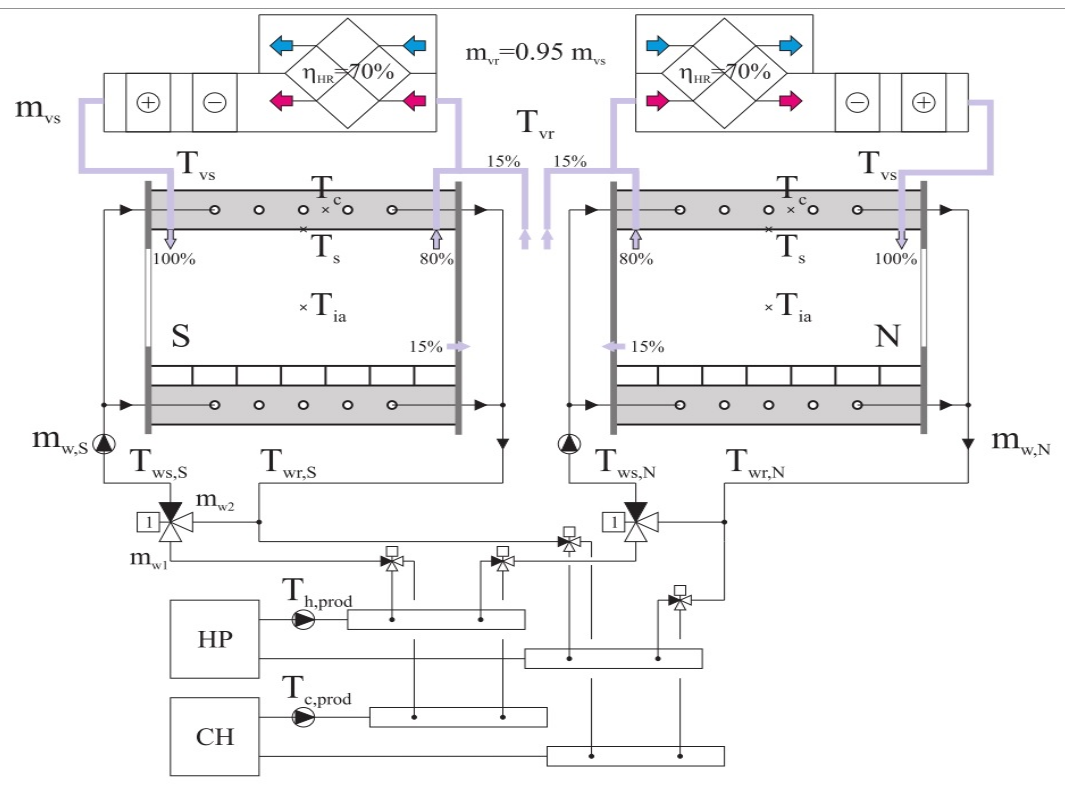

Figure 11: Schematic layout of the hydronic and aereolic installation in the reference case building. Source: Reprinted with permission from (7). 


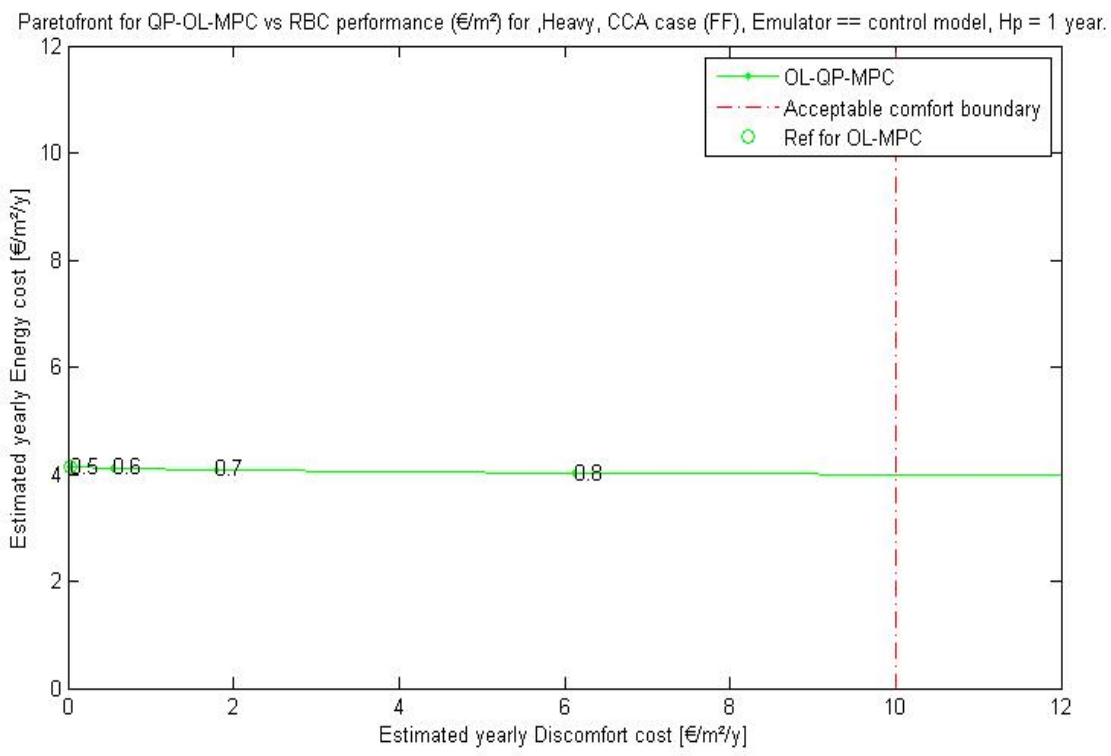

Figure 12: Simulated energy cost and discomfort cost for different weighting parameter values $\alpha$ while applying the OL-MPC controller on the controller model itself. The best economical trade-off is reached at an alfa value of 0.5 This parameters was used for the tuned OL-MPC. Operation in the area beyond $\left[10 / \mathrm{m}^{2} / y\right]$ discomfort (on the right of the dashed line, marked in red) are typically considered unacceptable. . 